\title{
Comprehensive Analysis of Prognostic Value and Immune Infiltration of Kindlin Family Members in Non-Small Cell Lung Cancer
}

\section{Xiaoshan Su}

Second Affiliated Hospital of Fujian Medical University

Ning Liu

Fuzhou Pulmonary Hospital of Fujian

\section{Weijing Wu}

Second Affiliated Hospital of Fujian Medical University

ZHIXING ZHU

Second Affiliated Hospital of Fujian Medical University

Yuan Xu

Second Affiliated Hospital of Fujian Medical University

Feng He

Fuzhou Pulmonary Hospital of Fujian

\section{Xinfu Chen}

Fuzhou Pulmonary Hospital of Fujian

Yiming Zeng ( $\nabla$ ymzeng08@163.com )

Second Affiliated Hospital of Fujian Medical University

\section{Research Article}

Keywords: Kindlins, Non-small cell lung cancer, Bioinformatics, Prognosis

Posted Date: February 4th, 2021

DOI: https://doi.org/10.21203/rs.3.rs-156779/v1

License: (c) (1) This work is licensed under a Creative Commons Attribution 4.0 International License. Read Full License 


\section{Comprehensive Analysis of Prognostic Value and Immune Infiltration of Kindlin Family}

2 Members in Non-Small Cell Lung Cancer

3 Xiaoshan $\mathrm{Su}^{1 \dagger}$, Ning $\mathrm{Liu}^{2 \dagger}$, Weijing $\mathrm{Wu}^{1}$, Zhixing $\mathrm{Zhu}^{1,3}$, Yuan $\mathrm{Xu}^{1}$, Feng $\mathrm{He}^{2}$, Xinfu $\mathrm{Chen}^{2}$,

$4 \quad$ Yiming Zeng ${ }^{1 *}$

5 1. Department of Pulmonary and Critical Care Medicine, the Second Affiliated Hospital of

6 Fujian Medical University, Respirology Medicine Centre of Fujian Province, Quanzhou,

7 China

8 2. Department of Thoracic Surgery, Fuzhou Pulmonary Hospital, Fuzhou, China

9 3. Department of Pathology and Biomedical Science, University of Otago, Christchurch, New

10 Zealand

11 †These authors contributed equally to this work. 
Background: Kindlin Family Members have been reported to be aberrantly expressed in various human cancer types and involved in tumorigenesis, tumor progression, and chemoresistance. However, their roles in non-small cell lung cancer (NSCLC) remain poorly elucidated.

Methods: We analyzed the prognostic value and immune infiltration of Kindlins in NSCLC through Oncomine, GEPIA, UALCAN, CCLE, Kaplan-Meier plotter, cBioPortal, TIMER, GeneMANIA, STRING, and DAVID database. Additionally, the mRNA expression levels of Kindlins were verified in 30 paired NSCLC tissues and NSCLC cell lines by real-time PCR.

Results: The expression level of FERMT1 was remarkably increased in NSCLC tissues and

NSCLC cell lines, while FERMT2 and FERMT3 were reduced. Kindlins expressions were associated with individual cancer stages and nodal metastasis status. We also found that higher expression level of FERMT1 was obviously correlated with worse overall survival (OS) in patients with NSCLC, while higher FERMT2 was strongly associated with better overall survival (OS) and first progression (FP). Additionally, the expression of FERMT2 and FERMT3 were obviously correlated with the immune infiltration of diverse immune cells. Functional enrichment analysis has shown that Kindlins may be significantly correlated with intracellular signal transduction, ATP binding and the PI3K-Akt signaling pathway in NSCLC. 
37 Conclusions: The research provides a new perspective on the distinct roles of Kindlins in NSCLC and likely has important implications for future novel biomarkers and therapeutic targets in NSCLC.

40 Keywords: Kindlins; Non-small cell lung cancer; Bioinformatics; Prognosis

41

42

43

44

45

46

47

48

49

50

51

52

53

54 
Lung cancer is known to the most common cancer and the leading cause of cancer mortality, causing approximately $1,760,000$ deaths worldwide each year[1]. Non-small cell lung cancer (NSCLC, approximately $15 \%$ of all cases) includes lung adenocarcinoma (LUAD), lung squamous cell carcinoma (LUSC) and lung large cell carcinoma (LCC)[2]. Although gradual improvements in diagnosis and treatment therapies, the 5-year overall survival rate of NSCLC remains poor. ${ }^{[3-4]}$ Thus, there is a need to explore the mechanism underlying the tumorigenesis and progression of NSCLC and to find novel biomarkers with high sensitivity and specificity. The Kindlin family members are newly discovered focal adhesion proteins consisting of three members (FERMT1, FERMT2 and FERMT3) that share a conserved FERM domain-containing three subdomains (F1, F2 and F3) with an inserted pleckstrin homology domain. Kindlins play crucial roles in various biological activities, including integrin activation, cell adhesion, migration, cell-cell junctions, and differentiation.[5-8] In addition, extensive research has revealed that Kindlins aberrant expressions in various human cancer types and these dysregulations are involved in tumorigenesis, tumor progression, and chemoresistance[9, 10]. Remarkably, Kindlins may function as tumor promoters or suppressors depending on the cancer type. For instance, FERMT2 severs as a tumor-promoting role in gastric cancer[11], pancreatic cancer[12, 13] and breast cancer[14, 
15], but as a tumor-suppressive role in epithelial ovarian cancer[16] and colorectal cancer[17].

Weinstein et al. reported a higher mRNA expression of FERMT1 in lung cancer tissues[18].

Besides, Zhan et al. also found that FERMT1 and FERMT2 had different expressions in lung cancer cells. FERMT1 was highly expressed in NSCLC, especially in LUSC. On the other hand, FERMT2 was highly expressed in LCC and weakly expressed in LUAD and LUSC.[19] Additionally, it was it was revealed that the expression of FERMT3 was reduced in lung cancer [20]. However, the series of research only reported the expression levels of Kindlins, and few studies have been conducted on the prognosis and mechanism of Kindlins.

Rapid advances in online platforms and various databases have contributed to the widely used bioinformatics analysis in the field of cancer research. Up to date, bioinformatics analysis has not been adequately used to investigate the roles of Kindlins in NSCLC. Hence, the present study investigated the role of individual kindlins in NSCLC using RT-PCR combined with bioinformatics. In this study, we investigated the roles of kindlin family members in NSCLC using bioinformatics combined with RT-PCR. We aim to investigate the prognostic value and immune infiltration of Kindlins in NSCLC, providing new clues for early diagnosis, prognostic judgments and individualized treatments for NSCLC patients. 


\section{Expression Database of Kindlins in NSCLC}

Oncomine database (http://www.oncomine.org) is a comprehensive cancer microarray database based on 86733 samples from 715 databases for gene transcriptome analysis in different cancers[21]. The screening and data mining conditions included: "Gene: FERMT1, FERMT2 and FERMT3”; “Analysis Type: Cancer vs. Normal Analysis"; "Cancer Type: Lung Cancer"; "Data Type: mRNA"; "p-Value < 0.05"; "Fold change:2"; "Gene rank: top 10\%". GEPIA database (http://gepia.cancer-pku.cn/) is a recently developed interactive online platform, applying for analyzing the RNA sequencing (RNA-Seq) expression based on over 9,000 tumors from the TCGA and 8,000 normal samples from the GTEx[22]. GEPIA was used to analyze Kindlins expression and tumor stage in LUAD and LUSC. UALCAN is a user-friendly web that provides publicly available cancer transcriptome data based on TCGA database[23]. UALCAN was used to confirm the association between mRNA expressions of Kindlins in NSCLC and clinicopathological parameters. CCLE (www.broadinstitute.org/ccle) provides the public information on gene expression, chromosome copy number and mutation profile of 947 human cancer cells[24]. In our research, we mainly use it to verify the expression levels of FERMT1, FERMT2 and FERMT3 in different kinds of cancer cell lines. 


\section{Kaplan-Meier plotter} Kaplan Meier plotter (http://kmplot.com/analysis) aims to discover and validate survival

111 biomarkers based on a meta-analysis from 11k samples from 20 different cancer types[25, 26].

112 In the study, we used the KM plotter database from a set of 1,926 lung cancer samples to assess Kindlins’ prognostic values, including overall survival (OS), first progression (FP) and

114 post-progression survival (PPS). Moreover, we evaluated the associations of the Kindlins with various clinical parameters of NSCLC, including the histology, clinical stages, gender and smoking history.

\section{cBioPortal}

multilayer cancer genome data from over 5,000 tumors from 20 different cancer studies[27, 28]. According to cBioPortal's online instructions, the Kindlins gene alterations information in amplifications, deep deletions and multiple alterations. 
127 the tumor-infiltrating immune cells from 32 cancer types[29]. In our research, Spearman

correlation was used in the gene module to explore the correlation between the expression of Kindlins and immune infiltration, including tumor purity and six types of immune cells (B cells, CD8+T cells, CD4+ T cells, macrophages, neutrophils, and dendritic cells).

\section{GeneMANIA and STRING database}

GeneMANIA (http://www.genemania.org) is a useful and flexible prediction server, displaying a functional interaction network to explore the association between genes and data sets[30]. In the current study, we used GeneMANIA to analyze the relationships between Kindlins concerning the co-expression, co-localization, physical interactions, pathway, genetic interactions, prediction, and shared protein domains.

STRING (https://string-db.org/) is an online database, predicting protein-protein interactions network in terms of direct (physical) and indirect (functional) associations[31]. In this study, we constructed the protein-protein interactions network of Kindlins using and the selection criteria: "organism: Homo sapiens"; "the minimum required interaction score > 0.4"; "the max number of interactors: $20 "$

\section{DAVID database}



platform, providing integrative and systematic annotations of biological functions from a series of genes/proteins[32, 33]. We applied the DAVID database (version 6.8) for gene ontology (GO) terms analysis and Kyoto Encyclopedia of Genes and Genomes (KEGG) pathways enrichment analysis of Kindlins and their related proteins. GO terms covers three aspects: biological processes, cellular components and molecular functions. The p-Value < 0.05 was set as a criterion and regarded as significant enrichment.

\section{Lung tissue samples}

In this study, between July 2019 and December 2019, thirty pairs of NSCLC tissue and adjacent normal tissues were collected from the Fuzhou Pulmonary Hospital, China. These tissues were used to detect the expression level of Kindlins mRNA by quantitative real-time PCR (RT-PCR). The collection and use of the samples were approved by the ethics committee of the Second Affiliated Hospital of Fujian Medical University. The approval number is 2019 (ethical research review)-207. 
were obtained from Procell life science and Technology Co., Ltd (Wuhan, China). Human

bronchial epithelial cells (BEAS-2B) were obtained from FenghBio Co., Ltd. (Changsha, China). All the cell lines were cultured at RPMI-1640 medium (GIBCO, Los Angeles, CA, USA) with $10 \%$ fetal bovine serum (FBS, Gibco) and grown in a humidified incubator at $37^{\circ} \mathrm{C}(5 \% \mathrm{CO} 2)$ environment.

\section{RT-PCR analysis}

Total RNA in tissues or cells was extracted using TRIzol reagent (Invitrogen) and cDNA was synthesized using Primescript RT Reagent (Takara Bio Inc., Japan). PCR amplification was performed using the SYBR Green PCR kit (Takara Bio Inc., Japan) in a 7500 PCR system (Thermo Fisher Scientific), and GAPDH was used as an endogenous control. The relative quantification analysis was performed using the comparative CT method. The following PCR primers were used:

FERMT1 forward, 5'-TTGAAGATGGTGAGGTTGCGAGTC-3'

FERMT1 reverse, 5'-GGGTTGGCTGAATGCGAGGATG-3'

FERMT2 forward, 5'-TGGCTCTGGACGGGATAAGGATG-3'

FERMT2 reverse, 5'-TTTGTGCTGAGGGGTGAACTGAAG-3'

FERMT3 forward, 5'-ACTGCACCGAGGAGGAGATGATG-3' 


\section{Statistical analysis}

RT-PCR was performed in triplicate. The data were analyzed using the GraphPad Prism

NSCLC tissue samples tissues and adjacent normal tissues. $\mathrm{P}<0.05$ was considered a statistically significant difference. 


\section{Results}

\section{Differential Expression of Kindlins in NSCLC Patients and Cell Lines}

We first used ONCOMINE database to explore the expression of Kindlins in NSCLC.

Multiple datasets showed that the mRNA expression level of FERMT1 was significantly

increased in NSCLC tissues, while FERMT2 and FERMT3 were reduced in NSCLC vs.

normal tissues (Figure 1A and Table 1,). These database include the Hou's dataset[34], Su dataset[35], Okayama dataset[36], Wachi dataset[37], Selamat's dataset[38], Landi's dataset[39], and Stearman's dataset [40]. Moreover, Meta-analysis of Kindlins genes expression in NSCLC studies from Oncomine databases were consistent with the above results (Figure 1B). Then, we used the GEPIA and UALCAN dataset to further confirm these findings. The results indicated that the expression level of FERMT1 was overexpressed in LUAD and LUSC tissues than in normal tissues, while FERMT2 and FERMT3 were

211 decreased inversely (Figure 1C-E). We also used CCLE to explore expression levels of Kindlins in NSCLC cell lines. As presented in Figure 2A, the expression level of FERMT1 was higher in NSCLC cell lines and FERMT3 was downregulated. These results were consistent with those from Oncomine, GEPIA and UALCAN dataset. Different from these results, FERMT2 expression was increased in NSCLC, which requires further examination. 
217 determined in 30 paired NSCLC tissues and NSCLC cell lines via RT-PCR. As expected, the results indicated that the FERMT1 expression was upregulated, while FERMT2 and FERMT3

219 were down-regulated in the tissue samples from 30 NSCLC cases $(\mathrm{P}<0.05$, Figures $2 \mathrm{~B})$. Furthermore, the expression level of FERMT1 was highly expressed in NSCLC cell lines (A549, SPCA-1 and H1299) compared with normal cell lines (BEAS-2B) (P <0.05, Figure 2C). For FERMT2 and FERMT3, the mRNA expression levels were reduced in NSCLC cell lines $(\mathrm{P}<0.05$, Figures $2 \mathrm{C})$. In conclusion, FERMT1 was prominently upregulated while FERMT2 and FERMT3 were down-regulated in NSCLC patients and NSCLC cell lines.

Association between the Kindlins expression and clinicopathological parameters in

\section{NSCLC}

We then further investigated the associations between the Kindlins mRNA expression and tumor stages in NSCLC using GEPIA and UALCAN dataset. As presented in Figure 3A, the expression levels of FERMT1 and FERMT2 were remarkably distinct in different tumor stages of LUAD and LUSC, while there was no significance between FERMT3 and different tumor stages. We then explored the expression of Kindlins in distinct individual cancer stages and nodal metastasis status of LUAD and LUSC using UALCAN dataset. It was shown that 
of LUAD and LUSC, while FERMT2 and FERMT3 genes were downregulated (Figure 3B).

Besides, the expression levels of Kindlins were significantly associated with nodal metastasis

status (Figure 3C). These data suggested that FERMT1, FERMT2 and FERMT3 might play important parts in the tumorigenesis and progression of NSCLC.

\section{Prognostic analysis of Kindlins in patients with NSCLC}

higher FERMT2 expression was strongly associated with better long-term OS $(\mathrm{HR}=0.76$,

$\log$-rank $\mathrm{P}=1.4 \mathrm{e}-05)$ and $\mathrm{FP}(\mathrm{HR}=0.82$, log-rank $\mathrm{P}=0.04)$ (Figure 4). Besides, patients with

high FERMT3 mRNA levels had longer OS, but the difference was not significant $(\mathrm{HR}=0.86$,

in NSCLC patients with stage 1, suggesting that FERMT2 might act as a prognostic role in 


\section{Alterations of Kindlins in patients with NSCLC}

We then explored the gene alterations of Kindlins in NSCLC using the cBioPortal database.

The Kindlins gene alterations were analyzed in 31 cancer studies, which included 10,931

samples. The results showed that gene alterations in Kindlins were present in different types of NSCLC, including LUSC and LUAD, compared with other cancer types (Figure 5A).

Furthermore, alterations frequencies and alterations types of Kindlins were determined in

$5.45 \%$ of the 3025 cases of NSCLC had mutations, amplifications, deep deletions and

gene alterations for FERMT1 and FERMT2, whereas gene mutations made up the majority of genetic alterations for FERMT3 (Figure 5C).

\section{Correlation between Kindlins expression and immune infiltration in NSCLC}


using TIMER. We found that FERMT3 expression is significantly negatively associated with

CD4+ T cell, Macrophage, Neutrophil, and Dendritic cell in LUAD and LUSC as presented in

Figure 6C. Additionally, FERMT2 expression showed a similar relationship with tumor purity and the infiltration of CD8+ T cell, CD4+ T cell, Macrophage, Neutrophil, and Dendritic cell in LUAD and LUSC (Figure 6B). However, the correlation strengths between FERMT1 expression and the six types of immune cells were all relatively weaker (Figure 6A).

\section{Interaction network of Kindlins at the gene and protein levels}

Moreover, the gene-gene interaction network of Kindlins is generated through

GeneMANIA. As presented in Figure 7A, three central nodes implying Kindlins were surrounded by 20 nodes implying genes that were closely associated with Kindlins concerning physical interactions, co-expression, predictions, co-localization, pathway, genetic interactions and shared protein domains. The top five genes showing the greatest connections with the Kindlins contained FBLIM1 (filamin binding LIM protein1), PARVB (Parvin beta), 
interactions and predictions. Moreover, the functional analysis suggested that these genes

were strongly correlated with focal adhesion, integrin activation, cell junction organization and cell-substrate adherens junction. (FERMT1, FERMT2, and FERMT3) and 20 functional partners with the highest confidence scores (score $>0.900$, Figure 7B). The top five proteins concerning the greatest associations with the Kindlins contained FBLIM1(filamin binding LIM protein1), CDC5L (cell division cycle 5-like protein), ILK (integrin-linked protein kinase), PICALM results indicated that the main biological processes involved the PPI network were platelet degranulation, cell-substrate adhesion, cell adhesion, substrate adhesion-dependent cell spreading, and cell-matrix adhesion, and the main KEGG pathways were focal adhesion, platelet activation, complement and coagulation cascades, amoebiasis and ECM-receptor interaction.

\section{Functional enrichment analysis of Kindlins in NSCLC}


BCL2, GSK3A, GSK3B, TSC2, STAT3, AKT1, AKT2, AKT3, TSC1, AXL, PDCD1, MAPK1,

CASP3, PXN, FOXO3 and NOTCH $(\mathrm{p}$-Value $<0.05)$ were primarily correlated with genomic

alterations of Kindlins in NSCLC. Furthermore, enrichment analysis of GO terms and KEGG

pathways of these differential proteins was performed using DAVID (S3-6). The top 5 results

of GO terms analysis with the terms of biological process analysis were intracellular signal

transduction, cell surface receptor signaling pathway, cellular response to chemical stimulus,

programmed cell death and cell death (Figure 8A). For cellular components, Kindlins were

significantly enriched in the cytosol, nucleoplasm, mitochondrion, actin cytoskeleton and

actin filament (Figure 8B). Molecular function mainly included ATP binding, protein

serine/threonine kinase activity, kinase activity, transcription factor activity and

showed that, based on gene count and P-value, the PI3K-Akt signaling pathway is regarded to

be the most significant for Kindlins in NSCLC. Additionally, KEGG pathway enrichment 


\section{Discussion}

Kindlins belong to the newly discovered focal adhesion proteins. Previous studies have

revealed that Kindlins were aberrantly expressed in various human cancer types and these

dysregulations have involvement in various biological processes, including proliferation, epithelial-mesenchymal transition, migration, invasion, and metastasis[9, 10]. However, the complex roles and significance of Kindlins in NSCLC have not been well elucidated. This study for the first time explored the expression patterns, prognostic value, immune infiltration and potential functions of Kindlins in NSCLC. We found Kindlins could play a tumor-promoting or tumor-suppressive role in NSCLC development. Compared with normal tissue, FERMT1 was highly expressed and correlated a worse prognosis in patients with

NSCLC, while FERMT2 had an opposite effect. Besides, the expression level of FERMT3 was reduced in patients with NSCLC and higher FERMT3 mRNA levels had longer OS, although the prognosis was not statistically significant. Additionally, a genetic alteration rate of Kindlins (5.9\%) was found in patients with NSCLC. These results indicated that Kindlins plays critical roles in NSCLC. 
Prior studies have revealed that FERMT1 is overexpressed and acts as a tumor promoter in various cancer types, including breast[42, 43], colon[44], pancreatic[45], and 345 hepatocellular[46] cancer. Mechanistically, several interesting linkages have indicated that FERMT1 may be involved in tumor occurrence and development by regulating the transforming growth factor-beta (TGF $\beta$ ) signaling[43, 44, 47]. It was reported that FERMT1 was overexpressed in NSCLC and inhibited tumor epithelial-mesenchymal transition, growth and invasion [18][19]. Adversely, Sin et al. reported that FERMT1 expression was related to a poor prognosis in lung and breast adenocarcinoma, and FERMT1 may be a strategy for inhibiting metastasis[43]. A similar tumorigenic effect of FERMT1 in NSCLC was also shown in our study. The expression of FERMT1 was remarkably increased in patients with NSCLC and NSCLC cell lines, and FERMT1 was significantly associated with tumor grade and nodal metastasis status. Moreover, the higher FERMT1 expression was significantly related to poor OS in NSCLC. These results suggested that FERMT1 might act as a potential therapeutic target in NSCLC. cells[41]. The potential role of FERMT2 in various types of cancer has been extensively explored. It has been suggested that FERMT2 acts either as a tumor promoter or suppressor in different cancers. Recent research has demonstrated that FERMT2 is overexpressed in many 
tumor types, including gastric[11], breast[14, 15], kidney[48], glioblastoma[49], pancreatic[12, 13], bladder[50], prostate[51], hepatocellular [52] and esophageal[53] cancer, and associated with tumor progression and poor prognosis. In addition, studies have shown that FERMT2 can down-regulate and inhibit tumor cell functions in epithelial ovarian cancer[16], colorectal cancer[17] and mesenchymal cancer cell[54]. In lung cancer, the expression pattern of FERMT2 in previous studies is controversial. Weinstein et al. found that the expression of FERMT2 did not observe a significant change in lung cancer[18]. Zhan et al. reported that FERMT2 was overexpressed in LCC, weakly expressed in LUAD and LUSC and promoted tumor invasion and growth in NSCLC[19]. The present study showed that FERMT2 was markedly decreased in NSCLC tissues and NSCLC cell lines. The previous result[19] was inconsistent with ours, which may be caused by the small sample size and different detection methods in different studies. Additionally, higher FERMT2 expression was strongly associated with long-term OS and FP in NSCLC, and FERMT2 was correlated with tumor grade and nodal metastasis status. Therefore, FERMT2 may be used as a prognostic biomarker for NSCLC. FERMT3, also known as Kindlin-3 and URP2, is mainly expressed in hematopoietic cells [55]. It was reported that FERMT3 was down-regulated and plays a tumor suppressor in many solid tumors including breast cancer, melanoma, as well as lung cancer[20]. Nevertheless, its 
function as a cancer suppressor or promoter is controversial. Previous research showed that FERMT3 was overexpressed in breast cancer and enhances cancer invasion and metastasis[56]. Moreover, Lu et al. found that FERMT3 was obviously upregulated in glioma, and knockdown of FERMT3 effectively suppressed glioma cell proliferation and chemoresistance[57]. Our study was consistent with the above that FERMT3 expression was significantly decreased in NSCLC, while the expression was not obviously associated with different tumor stages and OS in NSCLC patients. Hence, the prognostic significance of FERMT3 in NSCLC requires further investigation.

The tumor microenvironment (TME) serves an important role in tumor proliferation and

correlated with the immune infiltration of six immune cells (B cells, CD8+T cells, CD4+ T cells, macrophages, neutrophils, and dendritic cells) in LUAD and LUSC, suggesting that blockade therapy.

FERMT2 and FERMT3 may be potential biomarkers of NSCLC for immune checkpoint interacting genes and proteins are strongly correlated with integrin activation, cell junction, focal adhesion and cell adhesion. This suggests that Kindlins may be involved in the tumor 
397 progression by regulating integrin activation, cell junction, and focal adhesion. Enrichment 398 analysis indicated that the functions of Kindlins and their related differential proteins are 399 mainly associated with the intracellular signal transduction, cell surface receptor signaling 400 pathway, cellular response to chemical stimulus, programmed cell death and cell death in 401 NSCLC. These findings were consistent with previous studies that Kindlins serve important 402 roles as regulators of integrin inside-out signaling[59]. Furthermore, enrichment analysis 403 suggested that Kindlins were primarily correlated with the PI3K-Akt signaling pathway, 404 pathways in cancer, HIF-1 signaling pathway, insulin signaling pathway and mTOR signaling 405 pathway. These data suggest that Kindlins may modulate tumor development and progression 406 by regulating these signaling pathways in NSCLC, emphasizing their potential as 407 anti-NSCLC therapeutic targets.

408 There were some limitations to this study that need to be noted. First, our study was 409 primarily based on the transcriptional level, whereas protein expressions need further 410 investigation. Additionally, this study didn't carry out experiments on the biological 411 mechanisms of Kindlins in NSCLC. To address these issues, further in vitro and in vivo 412 studies need to validate these findings. 


\section{Conclusion}

416 In summary, we analyzed the prognostic value and immune infiltration of individual

417 Kindlins in NSCLC through RT-PCR combined with bioinformatics analysis. The expression 418 level of FERMT1 was significantly elevated in NSCLC tissues and NSCLC cell lines, while 419 the expression levels of FERMT2 and FERMT3 were reduced. Kindlins expressions are 420 significantly correlated with individual cancer stages and nodal metastasis status. We also 421 found that FERMT1 expression was significantly associated with short OS in NSCLC patients, 422 while higher FERMT2 expression was strongly associated with better OS and FP. In addition, FERMT2 and FERMT3 were significantly correlated with the immune infiltration.

424 Collectively, the research provides a new perspective on the distinct roles of Kindlins in 425 NSCLC and likely has important implications for future effective therapeutic targets in 426 NSCLC.

Ethics approval and consent to participate

The study protocol was approved by the ethics committee of the Second Affiliated Hospital of 
434 Not applicable.

435

\section{Availability of data and materials}

All methods were performed in accordance with the relevant guidelines and regulations;

Direct web links of datasets about; Oncomine: http://www.oncomine.org; GEPIA database:

http://gepia.cancer-pku.cn/;

UALCAN:

http://ualcan.path.uab.edu;

CCLE:

www.broadinstitute.org/ccle; Kaplan Meier plotter: http://kmplot.com/analysis;

cBioPortal :http://cbioportal.org; TIMER: https://cistrome.shinyapps.io/timer/; GeneMANIA :

http://www.genemania.org; STRING: https://string-db.org/; DAVID: https://david.ncifcrf.gov.

\section{Competing Interests}

445

The authors declare that there are no competing interests associated with the study.

\section{Funding}

The present study was supported by The National Key Research and Development Program of

Medical University (Grant number 2019QH2038). 


\section{Authors' contributions}

Xiaoshan Su, Ning Liu and Weijing Wu conceived and designed the study. Zhixing Zhu and

Yuan $\mathrm{Xu}$ prepared the figures and tables. Feng $\mathrm{He}$ and Xinfu Chen analyzed the data.

Xiaoshan Su and Ning Liu drafted the manuscript. Yiming Zeng provided fund support.

Yiming Zeng was involved in drafting the manuscript or revising it critically for important intellectual content. All authors read and approved the final manuscript.

\section{Abbreviations}

NSCLC, non-small cell lung cancer; LUAD, lung adenocarcinoma; LUSC, lung squamous cell carcinoma; LCC, lung large cell carcinoma; GEPIA, Gene Expression Profiling Interactive Analysis; cBioPortal, cBio Cancer Genomics Portal; STRING, Search Tool for the

Integrated Discovery; OS: overall survival; FP, first progression; PPS, post-progression survival; PPI ,protein-protein interaction; HR, hazard ratio; CI, confidence intervals. 
469 with different clinical parameters; Supplementary material 2: Proteomic data; Supplementary

470 material 3: GO Biological Process; Supplementary material 4: GO Cellular Component;

471 Supplementary material 5: GO Molecular Function; Supplementary material 6: KEGG

472 pathway.

473

474 Acknowledgements

475 Not applicable.

476

477

478

479

480

481

482

483

484

485

486 


\section{References}

488

489

490

491

492

493

494

495

496

497

498

499

500

501

502

503

504

1. Bray F, Ferlay J, Soerjomataram I, Siegel RL, Torre LA, Jemal A. Global cancer statistics 2018: GLOBOCAN estimates of incidence and mortality worldwide for 36 cancers in 185 countries. CA Cancer J Clin. 2018;68:394-424. doi:10.3322/caac.21492.

2. Osmani L, Askin F, Gabrielson E, Li QK. Current WHO guidelines and the critical role of immunohistochemical markers in the subclassification of non-small cell lung carcinoma (NSCLC): Moving from targeted therapy to immunotherapy. Semin Cancer Biol.

2018;52:103-9. doi:10.1016/j.semcancer.2017.11.019.

3. Duma N, Santana-davila R, Molina JR. Non-Small Cell Lung Cancer: Epidemiology, Screening, Diagnosis, and Treatment. Mayo Clin Proc. 2019;94:1623-40.

doi:10.1016/j.mayocp.2019.01.013.

4. Ettinger DS, Wood DE, Aggarwal C, Aisner DL. NCCN Guidelines Insights: Non-Small Cell Lung Cancer, Version 1.2020. J Natl Compr Cancer Netw. 2019;17:1464-72. doi:10.6004/jncen.2019.0059.

5. Meves A, Stremmel C, Gottschalk K, Fässler R. The Kindlin protein family: new members to the club of focal adhesion proteins. Trends Cell Biol. 2009;19:504-13. doi:10.1016/j.tcb.2009.07.006.

6. Li H, Deng Y, Sun K, Yang H, Liu J, Wang M, et al. Structural basis of kindlin-mediated 
integrin recognition and activation. Proc Natl Acad Sci. 2017;114:9349-54.

doi:10.1073/pnas.1703064114.

7. Sun Z, Costell M, Fässler R. Integrin activation by talin, kindlin and mechanical forces. Nat Cell Biol. 2019;21:25-31. doi:10.1038/s41556-018-0234-9.

8. Lai-Cheong JE, Parsons M, McGrath JA. The role of kindlins in cell biology and relevance to human disease. Int J Biochem Cell Biol. 2010;42:595-603.

doi:10.1016/j.biocel.2009.10.015.

9. Plow EF, Das M, Katarzyna, Bialkowska, Sossey-Alaoui K. Of Kindlins and Cancer.

Discov. 2016;4:1-15. doi:10.15190/d.2016.6.Of.

10. Zhan J, Zhang H. Kindlins: Roles in development and cancer progression. Int J Biochem

Cell Biol. 2018;98:93-103. doi:10.1016/j.biocel.2018.03.008.

11. Shen Z, Ye Y, Kauttu T, Seppänen H, Vainionpää S, Wang S, et al. Novel focal adhesion protein kindlin-2 promotes the invasion of gastric cancer cells through phosphorylation of integrin $\beta 1$ and $\beta 3$. J Surg Oncol. 2013;108:106-12. doi:10.1002/jso.23353.

12. Mahawithitwong P, Ohuchida K, Ikenaga N, Fujita H, Zhao M, Kozono S, et al. Kindlin-2 expression in peritumoral stroma is associated with poor prognosis in pancreatic ductal adenocarcinoma. Pancreas. 2013;42:663-9. doi:10.1097/MPA.0b013e318279bd66.

13. Yoshida N, Masamune A, Hamada S, Kikuta K, Takikawa T, Motoi F, et al. Kindlin-2 in 
pancreatic stellate cells promotes the progression of pancreatic cancer. Cancer Lett.

2017;390:103-14. doi:10.1016/j.canlet.2017.01.008.

14. Guo B, Gao J, Zhan J, Zhang H. Kindlin-2 interacts with and stabilizes EGFR and is required for EGF-induced breast cancer cell migration. Cancer Lett. 2015;361:271-81. doi:10.1016/j.canlet.2015.03.011.

15. Xue X, Li J, Wan W, Shi X, Zheng Y. Kindlin-2 could influence breast nodule elasticity and improve lymph node metastasis in invasive breast cancer. Sci Rep. 2017;7:1-10. doi:10.1038/s41598-017-07075-1.

16. Ren C, Du J, Xi C, Yu Y, Hu A, Zhan J, et al. Kindlin-2 inhibits serous epithelial ovarian cancer peritoneal dissemination and predicts patient outcomes. Biochem Biophys Res Commun. 2014;446:187-94. doi:10.1016/j.bbrc.2014.02.087.

17. Ren Y, Jin H, Xue Z, Xu Q, Wang S, Zhao G, et al. Kindlin-2 inhibited the growth and migration of colorectal cancer cells. Tumor Biol. 2015;36:4107-14. doi:10.1007/s13277-015-3044-8. 18. Weinstein EJ, Bourner M, Head R, Zakeri H, Bauer C, Mazzarella R. URP1 : a member of a novel family of PH and FERM domain-containing membrane-associated proteins is significantly over-expressed in lung and colon carcinomas. Biochim Biophys Acta. 2003;1637:207-16. doi:10.1016/S0925-4439(03)00035-8. 

suppressor function of Kindlin-3 in solid cancer. Oncotarget. 2014;5:8970-8985. doi:10.18632/oncotarget.2125.

21. Rhodes DR, Kalyana-Sundaram S, Mahavisno V, Varambally R, Yu J, Briggs BB, et al.

Oncomine 3.0: Genes, pathways, and networks in a collection of 18,000 cancer gene expression profiles. Neoplasia. 2007;9:166-80. doi:10.1593/neo.07112. normal gene expression profiling and interactive analyses. Nucleic Acids Res. 2017;45:W98-102. doi:10.1093/nar/gkx247.

23. Chandrashekar DS, Bashel B, Balasubramanya SAH, Creighton CJ, Ponce-Rodriguez I,

Expression and Survival Analyses. Neoplasia (United States). 2017;19:649-58. doi:10.1016/j.neo.2017.05.002. 
25. Gyorffy B, Surowiak P, Budczies J, Lánczky A. Online survival analysis software to assess the prognostic value of biomarkers using transcriptomic data in non-small-cell lung cancer. PLoS One. 2013;8. doi:10.1371/journal.pone.0082241.

26. Nagy Á, Lánczky A, Menyhárt O, Gyorffy B. Validation of miRNA prognostic power in hepatocellular carcinoma using expression data of independent datasets. Sci Rep. 2018;8:1-9. doi:10.1038/s41598-018-27521-y.

27. Cerami E, Gao J, Dogrusoz U, Gross BE, Sumer SO, Aksoy BA, et al. The cBio Cancer Genomics Portal: An open platform for exploring multidimensional cancer genomics data.

Cancer Discov. 2012;2:401-4. https://doi.org/10.1158/2159-8290.CD-12-0095.

28. Gao J, Aksoy BA, Dogrusoz U, Dresdner G, Gross B, Sumer SO, et al. Integrative analysis of complex cancer genomics and clinical profiles using the cBioPortal. Sci Signal. 2013;6. doi:10.1126/scisignal.2004088.

29. Taiwen Li, Jingyu Fan, Binbin Wang, Nicole Traugh, Qianming Chen, Jun S Liu B, Li and XSL. TIMER: A Web Server for Comprehensive Analysis of Tumor-Infiltrating Immune Cells. Physiol Behav. 2017;176:139-48.

30. Warde-Farley D, Donaldson SL, Comes O, Zuberi K, Badrawi R, Chao P, et al. The GeneMANIA prediction server: Biological network integration for gene prioritization and predicting gene function. Nucleic Acids Res. 2010;38 SUPPL. 2:214-20. 
doi:10.1093/nar/gkq537.

31. Szklarczyk D, Gable AL, Lyon D, Junge A, Wyder S, Huerta-Cepas J, et al. STRING v11:

Protein-protein association networks with increased coverage, supporting functional

discovery in genome-wide experimental datasets. Nucleic Acids Res. 2019;47:D607-13.

doi:10.1093/nar/gky1131.

32. Huang DW, Sherman BT, Lempicki RA. Bioinformatics enrichment tools: Paths toward the comprehensive functional analysis of large gene lists. Nucleic Acids Res. 2009;37:1-13.

doi:10.1093/nar/gkn923.

33. Huang DW, Sherman BT, Lempicki RA. Systematic and integrative analysis of large gene lists using DAVID bioinformatics resources. Nat Protoc. 2009;4:44-57.

doi:10.1038/nprot.2008.211.

34. Hou J, Aerts J, den Hamer B, van IJcken W, den Bakker M, Riegman P, et al. Gene

Expression-Based Classification of Non-Small Cell Lung Carcinomas and Survival Prediction.

PLoS One. 2010;5:e10312. doi:10.1371/journal.pone.0010312.

35. Su LJ, Chang CW, Wu YC, Chen KC, Lin CJ, Liang SC, et al. Selection of DDX5 as a novel internal control for Q-RT-PCR from microarray data using a block bootstrap re-sampling scheme. BMC Genomics. 2007;8:1-12. doi:10.1186/1471-2164-8-140.

36. Okayama H, Kohno T, Ishii Y, Shimada Y, Shiraishi K, Iwakawa R, et al. Identification 
of genes upregulated in ALK-positive and EGFR/KRAS/ALK-negative lung adenocarcinomas. Cancer Res. 2012;72:100-11. doi:10.1158/0008-5472.CAN-11-1403.

37. Wachi S, Yoneda K, Wu R. Interactome-transcriptome analysis reveals the high centrality of genes differentially expressed in lung cancer tissues. Bioinformatics. 2005;21:4205-8. doi:10.1093/bioinformatics/bti688.

38. Selamat SA, Chung BS, Girard L, Zhang W, Zhang Y, Campan M, et al. Genome-scale analysis of DNA methylation in lung adenocarcinoma and integration with mRNA expression. Genome Res. 2012;22:1197-211. doi:10.1101/gr.132662.111.

39. Landi MT, Dracheva T, Rotunno M, Figueroa JD, Liu H, Dasgupta A, et al. Gene expression signature of cigarette smoking and its role in lung adenocarcinoma development and survival. PLoS One. 2008;3. doi:10.1371/journal.pone.0001651.

40. Stearman RS, Dwyer-Nield L, Zerbe L, Blaine SA, Chan Z, Bunn PA, et al. Analysis of orthologous gene expression between human pulmonary adenocarcinoma and a carcinogen-induced murine model. Am J Pathol. 2005;167:1763-75. doi:10.1016/S0002-9440(10)61257-6.

41. Ussar S, Wang HV, Linder S, Fässler R, Moser M. The Kindlins: Subcellular localization and expression during murine development. Exp Cell Res. 2006;312:3142-51.

doi:10.1016/j.yexcr.2006.06.030. 
614 pulmonary breast cancer metastasis. Cancer Res. 2018;78:1484-96.

615 doi:10.1158/0008-5472.CAN-17-1518.

616 43. Sin S, Bonin F, Petit V, Meseure D, Lallemand F, Biche I, et al. Role of the focal

617 adhesion protein kindlin-1 in breast cancer growth and lung metastasis. J Natl Cancer Inst.

618 2011;103:1323-37. doi:10.1093/jnci/djr290.

619 44. Kong J, Du J, Wang Y, Yang M, Gao J, Wei X, et al. Focal adhesion molecule Kindlin-1

620 mediates activation of TGF- $\beta$ signaling by interacting with TGF- $\beta$ RI, SARA and Smad3 in

621 colorectal cancer cells. Oncotarget. 2016;7:76224-37. doi:10.18632/oncotarget.12779.

622 45. Mahawithitwong P, Ohuchida K, Ikenaga N, Fujita H, Zhao M, Kozono S, et al. Kindlin-1

623 expression is involved in migration and invasion of pancreatic cancer. Int J Oncol.

$624 \quad 2013 ; 42: 1360-6$. doi:10.3892/ijo.2013.1838.

625

46. Ma HX, Shu QH, Pan JJ, Liu D, Xu GL, Li JS, et al. Expression of Kindlin-1 in human

hepatocellular carcinoma and its prognostic significance. Tumor Biol. 2015;36:4235-41.

doi:10.1007/s13277-015-3060-8.

47. Kloeker S, Major MB, Calderwood DA, Ginsberg MH, Jones DA, Beckerle MC. The 
48. Yan M, Zhang L, Wu Y, Gao L, Yang W, Li J, et al. Increased expression of kindlin-2 is correlated with hematogenous metastasis and poor prognosis in patients with clear cell renal cell carcinoma. FEBS Open Bio. 2016;6:660-5. doi:10.1002/2211-5463.12063.

49. Ou Y, Zhao Z, Zhang W, Wu Q, Wu C, Liu X, et al. Kindlin-2 interacts with $\beta$-catenin and YB-1 to enhance EGFR transcription during glioma progression. Oncotarget. 2016;7:74872-85. doi:10.18632/oncotarget.12439.

50. Wu J, Yu C, Cai L, Lu Y, Jiang L, Liu C, et al. Effects of increased Kindlin-2 expression in bladder cancer stromal fibroblasts. Oncotarget. 2017;8:50692-703. doi:10.18632/oncotarget.17021.

51. Yang J rong, Pan T jun, Yang H, Wang T, Liu W, Liu B, et al. Kindlin-2 promotes invasiveness of prostate cancer cells via NF-kB-dependent upregulation of matrix metalloproteinases. Gene. 2016;576:571-6. doi:10.1016/j.gene.2015.11.005.

52. Lin J, Lin W, Ye Y, Wang L, Chen X, Zang S, et al. Kindlin-2 promotes hepatocellular carcinoma invasion and metastasis by increasing Wnt/ $\beta$-catenin signaling. J Exp Clin Cancer Res. 2017;36:1-14.

53. Wang P, Zhan J, Song J, Wang Y, Fang W, Liu Z, et al. Differential expression of Kindlin-1 and Kindlin-2 correlates with esophageal cancer progression and epidemiology. Sci China Life Sci. 2017;60:1214-22. doi:10.1007/s11427-016-9044-5. 
54. Shi X, Wu C. A suppressive role of mitogen inducible gene-2 in mesenchymal cancer cell invasion. Mol Cancer Res. 2008;6:715-24. doi:10.1158/1541-7786.MCR-07-2026. Immunol. 2014;3:37-42.

56. Sossey-Alaoui K, Pluskota E, Davuluri G, Bialkowska K, Das M, Szpak D, et al.

Kindlin-3 enhances breast cancer progression and metastasis by activating Twist-mediated angiogenesis. FASEB J. 2014;28:2260-71. doi:10.1096/fj.13-244004. cell proliferation and chemoresistance to temozolomide through integrin mediated Wnt signaling. Neurosci Lett. 2017;657:77-83. doi:10.1016/j.neulet.2017.07.057. Roles of MSCs in the Tumor Microenvironment: Interactions With Immune Cells and 


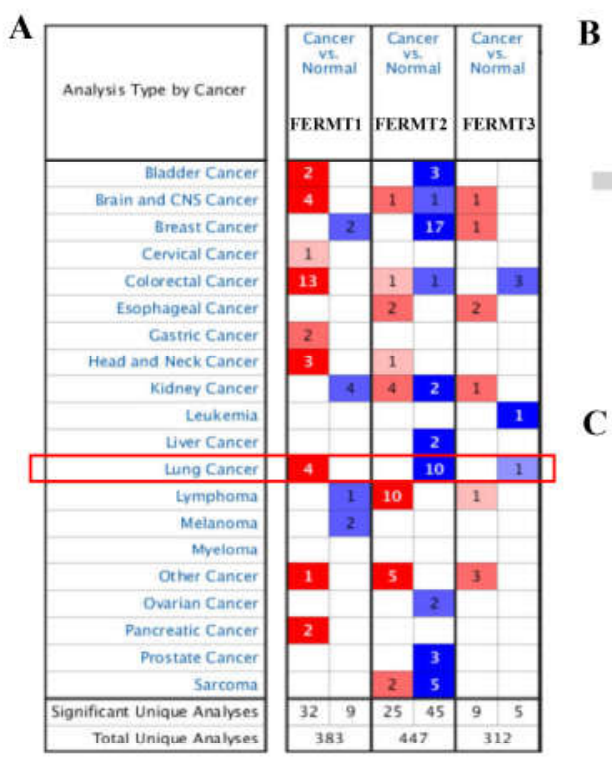

B
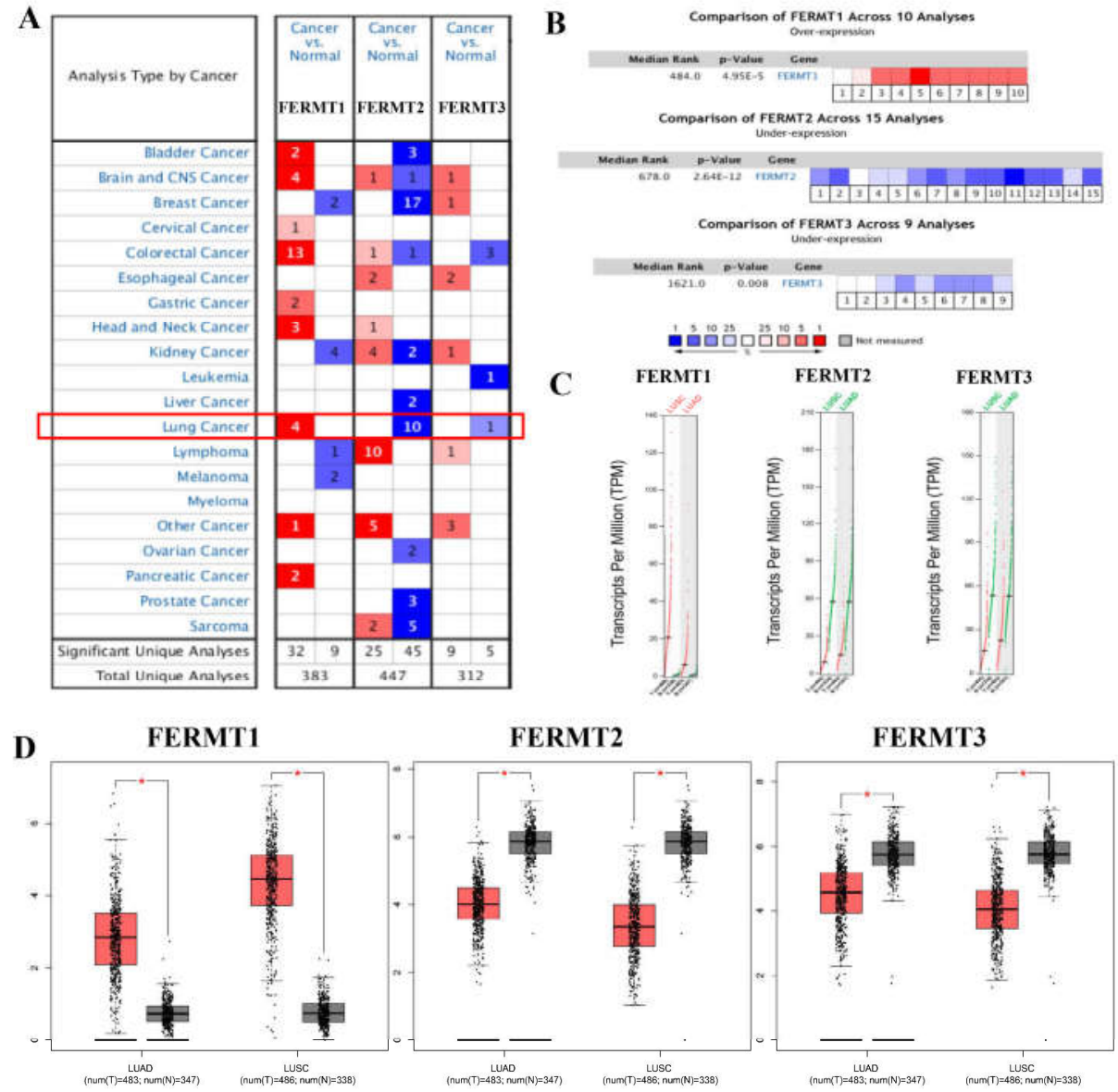

$\mathbf{E}$
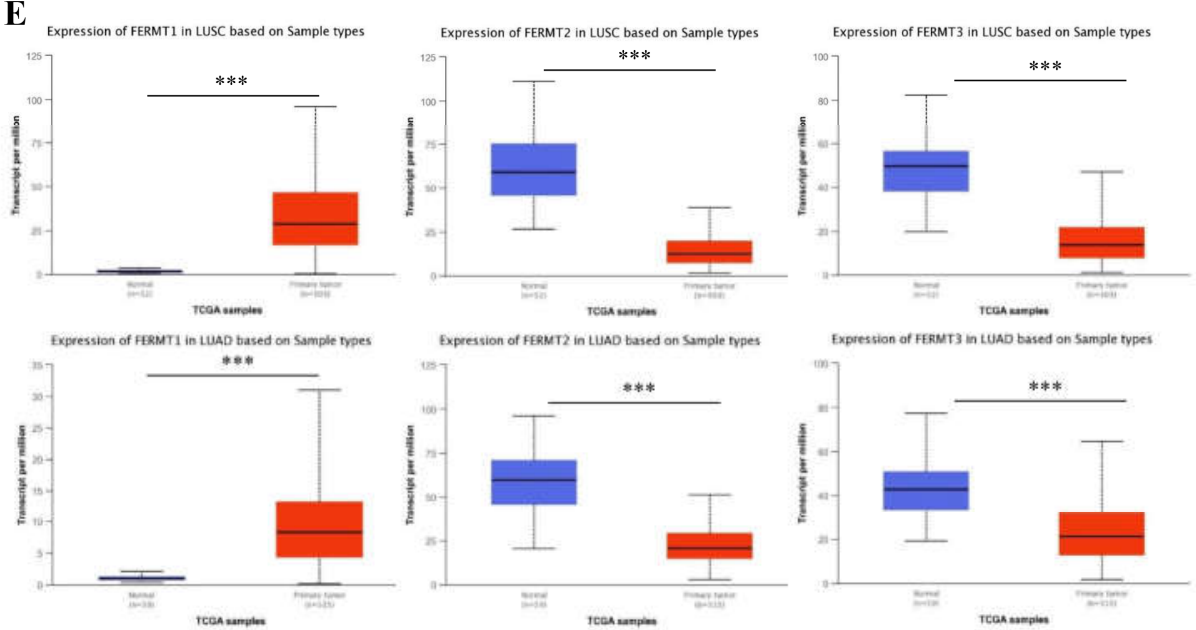

670 Fig.1. The expression of Kindlins in NSCLC. (A)The expression of Kindlins in different 
671 types of cancers compared with normal tissues (ONCOMINE). (B) Meta-analysis of

672 FERMT1, FERMT2 and FERMT3 mRNA expression in NSCLC from multiple Oncomine

673 databases. (C) The expression profile of Kindlins in LUAD and LUSD (GEPIA). Red trace,

674 tumor samples; green trace, normal samples; $\mathrm{T}$, tumor; $\mathrm{N}$, normal. (D) The expression

675 boxplots of Kindlins in LUSD and LUAD (GEPIA). A t-test was used to compare the

676 expression level differences between tumor and normal tissues $(\mathrm{P}<0.01)$. Y-axis represents

$677 \log 2(\mathrm{TPM}+1)$. Red box, tumor samples; black box, normal samples. T, tumor; N, normal. (E)

678 The relative expression of Kindlins in LUAD and LUSD (UALCAN).

679 

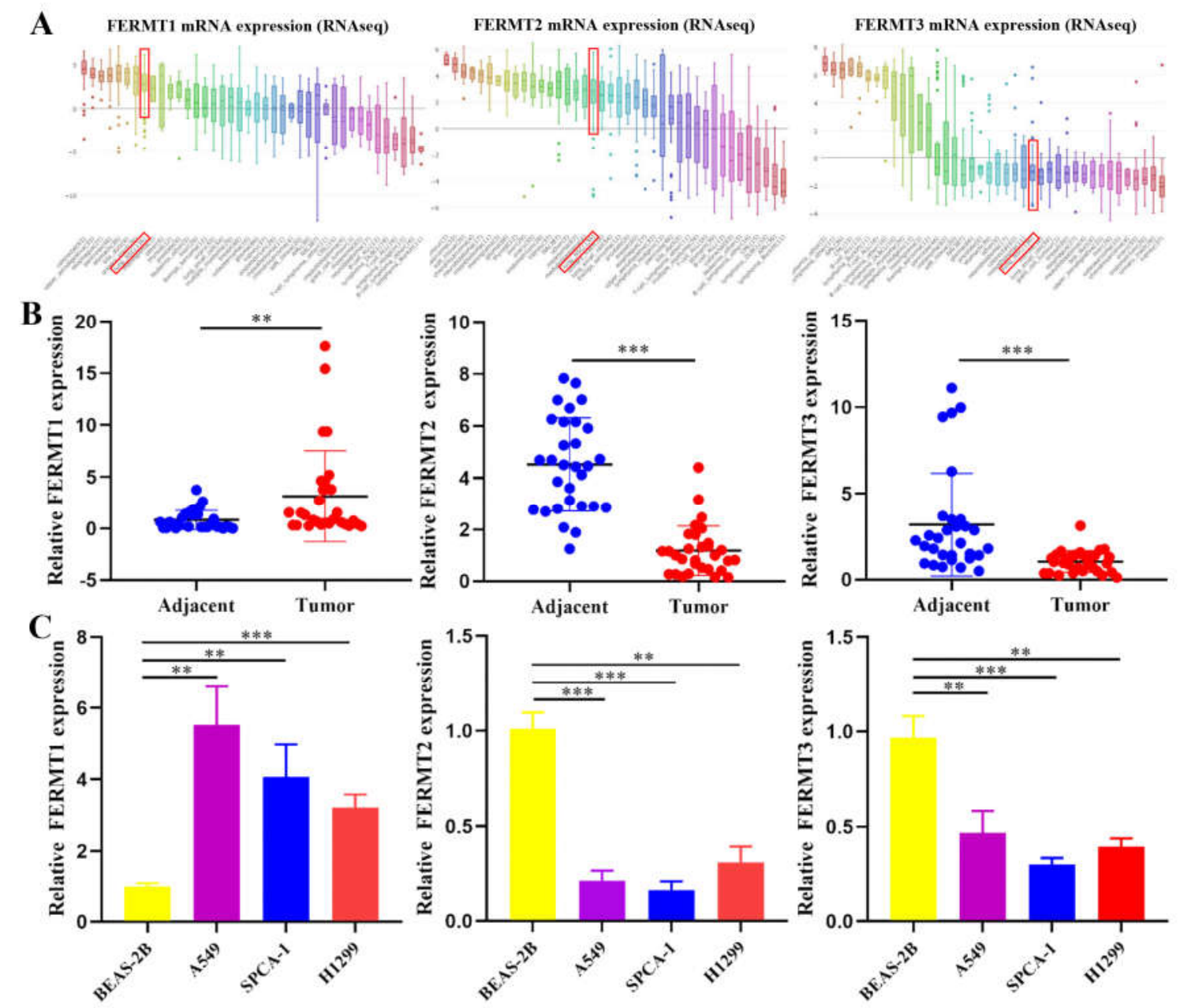

680
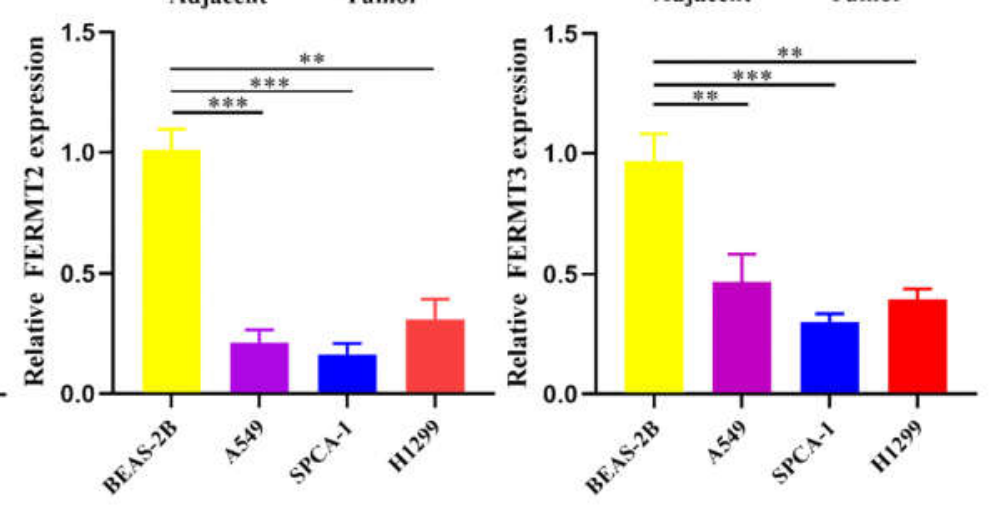

Fig. 2. The expression of Kindlins in NSCLC tissue and cell lines. (A) FERMT1, FERMT2 tissues in 41 NSCLC patients via RT-PCR assay. (C) Analysis of FERMT1, FERMT2 and FERMT3 expression in the NSCLC cell lines (A549, SPCA-1 and H1299) and one normal cell line (BEAS-2B) via RT-PCR assay. **p $<0.05 * * * \mathrm{p}<0.001$ 

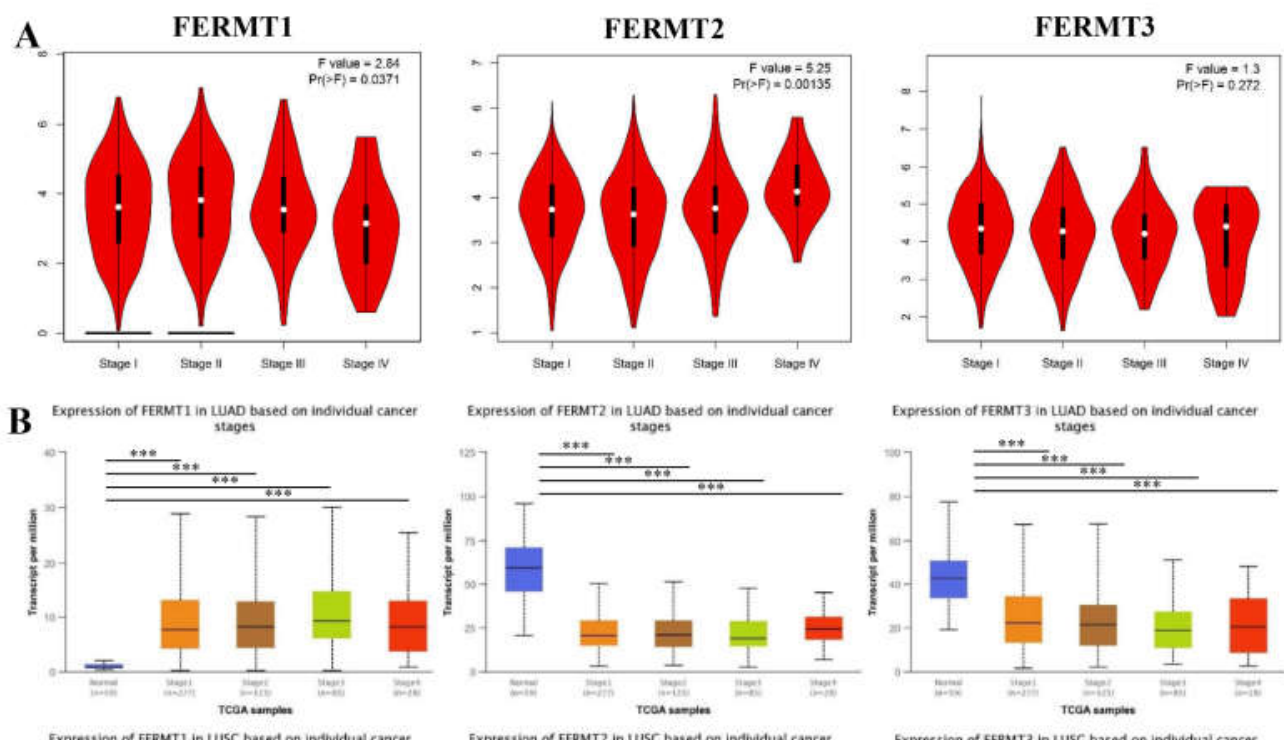

Expression of FERMT2 in LUAD based on individual cancer
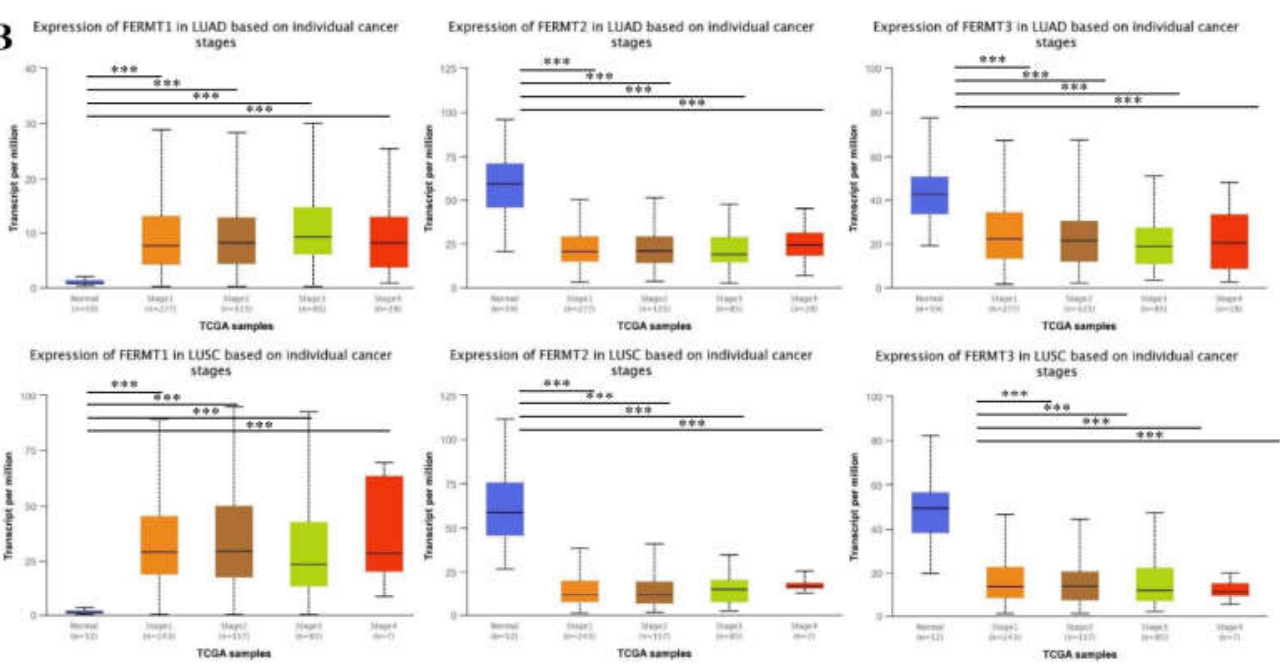

C
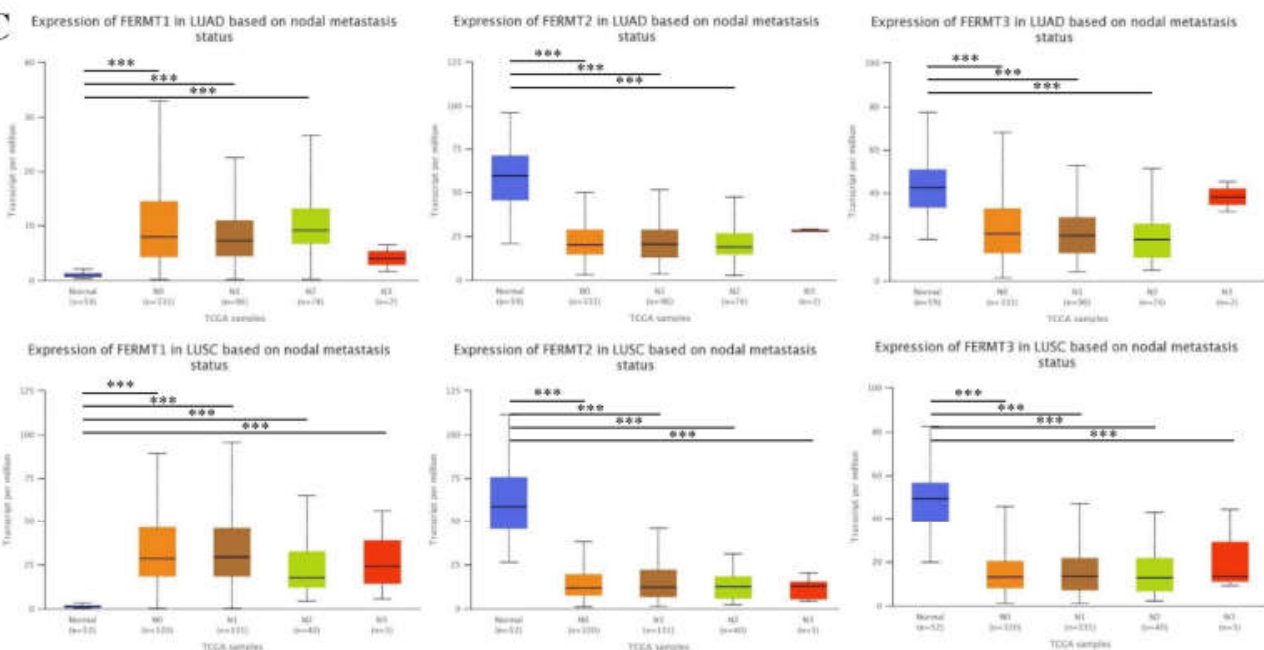

687

Fig. 3. Association between the Kindlins expression and clinicopathological parameters

689 in NSCLC. (A) Correlation between Kindlins expression and tumor stages in patients with

690 NSCLC (GEPIA). (B) The correlations between Kindlins mRNA expression and clinicopathological stages in LUAD and LUSD (UALCAN). (C) The correlations between 

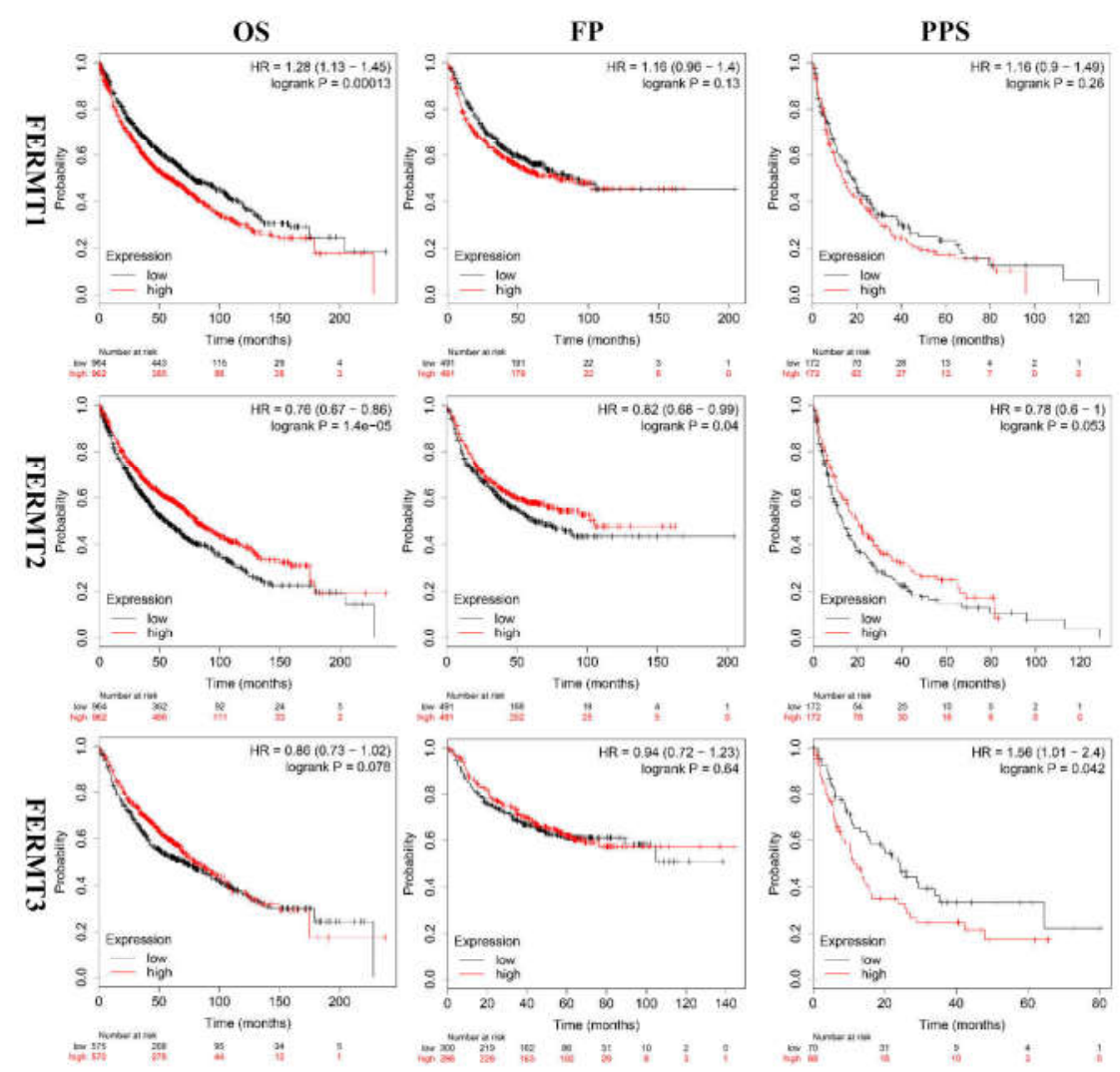

694

Fig.4. The prognostic value of the mRNA level of individual Kindlins in NSCLC patients

696 (Kaplan-Meier plotter). OS, overall survival; FP, first progression; PPS, post-progression survival. 


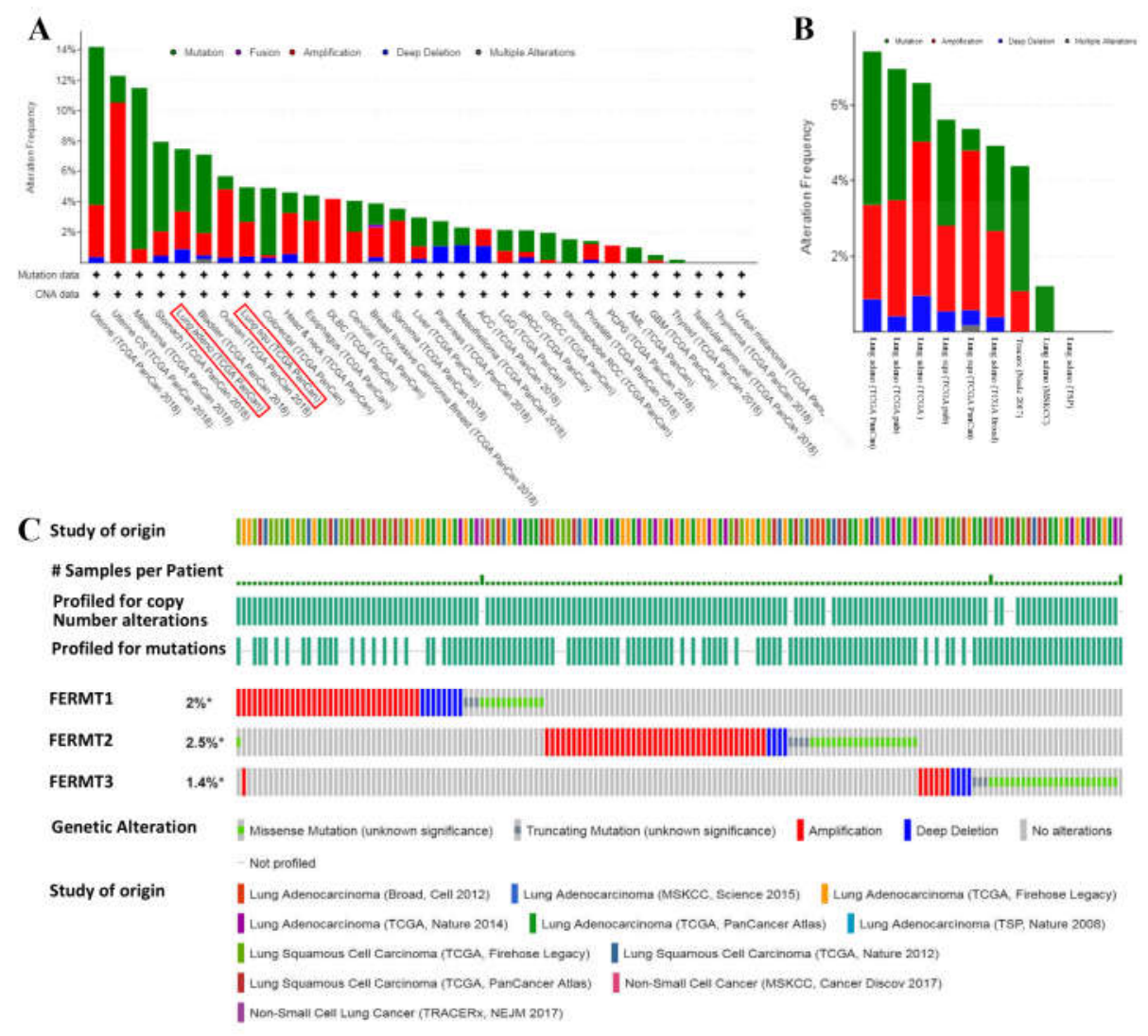

698

Fig.5. Analysis of Kindlins genetic alterations in NSCLC patients (cBioPortal). (A)

Genetic alternations frequencies of Kindlin family members in various carcinoma types.

Green, genetic mutations; purple, gene fusions; red, gene amplifications; blue, deep deletions; grey, multiple alterations. (B) Summary of Kindlin family genetic alterations in NSCLC. Alterations frequencies and alterations types of Kindlins were determined in 3897 patients / 4181 samples of 11 NSCLC studies. Green, genetic mutations; red, gene amplifications; blue, deep deletions; grey, multiple alterations. (C) OncoPrint visual summary of Kindlins alterations in NSCLC. green, missense mutations. gray, truncation mutation. Red, gene amplifications; blue, deep deletions. 

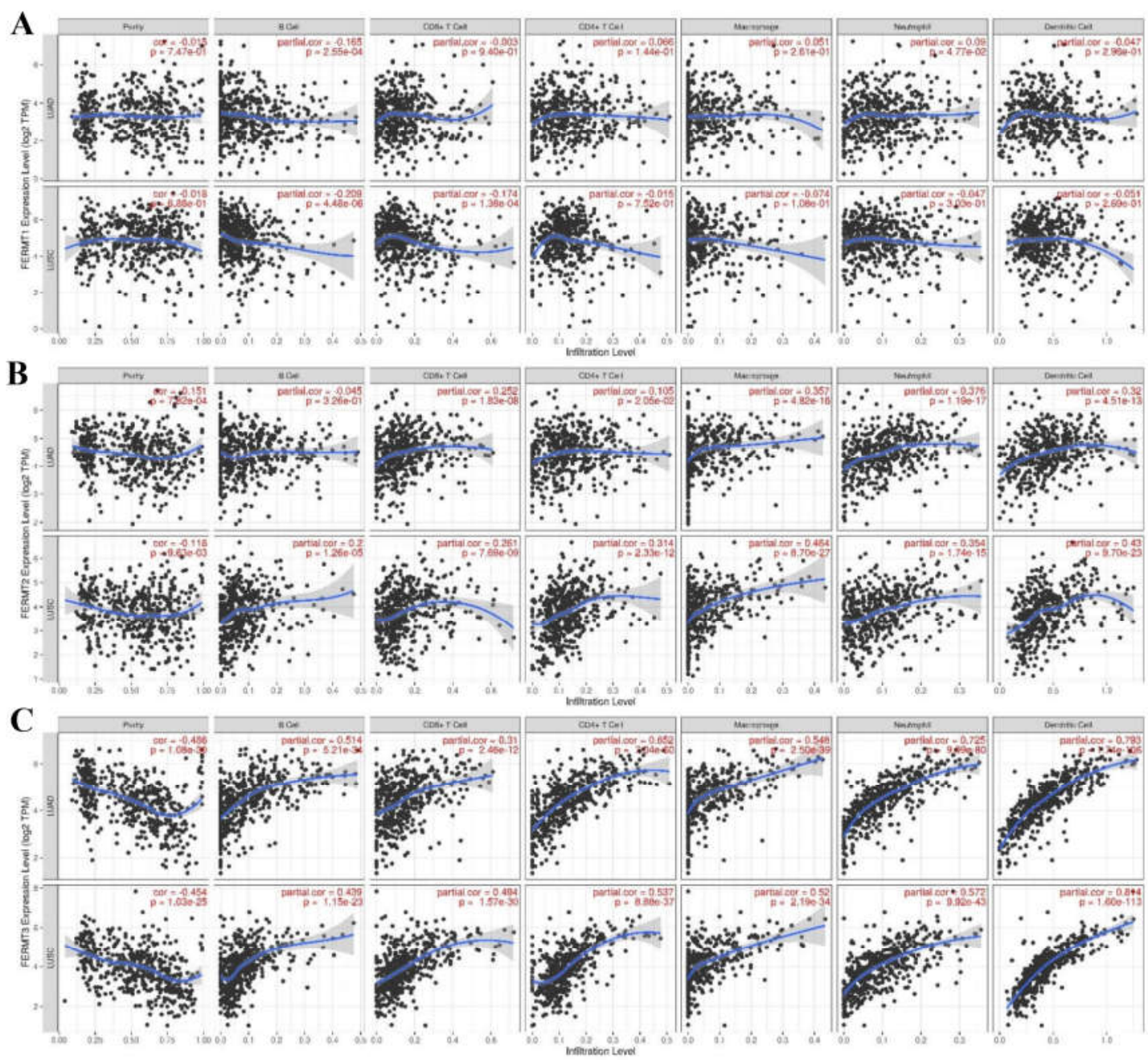

709

710 Fig.6. Association of Kindlins (FERMT1, FERMT2, and FERMT3) expression with

711 immune infiltration of immune cells in LUAD and LUSD patients (TIMER). Correlation

712 was analyzed using Pearson's Correlation. 

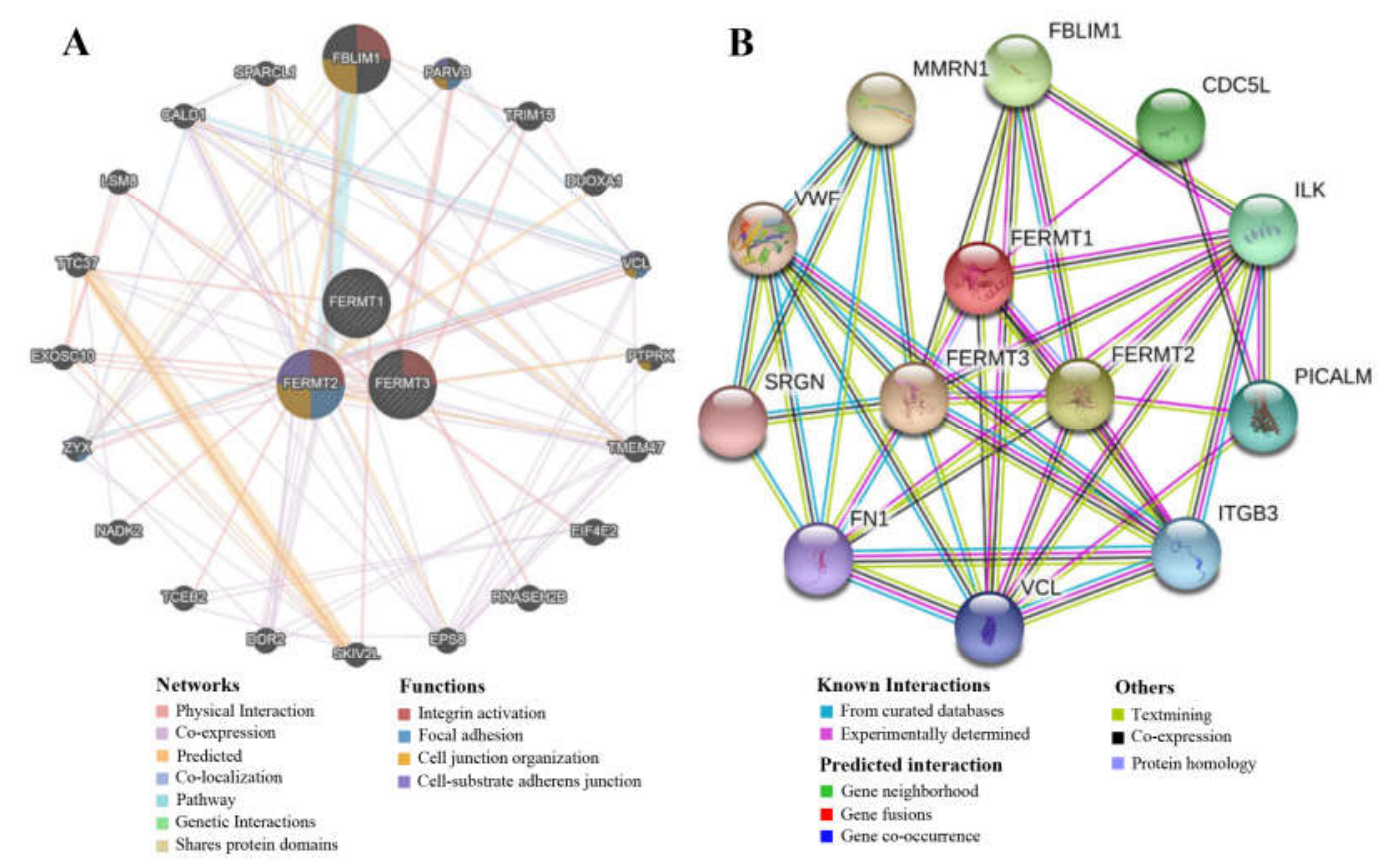

714 Fig.7. Interaction analysis of Kindlins family members. (A) Gene-gene interaction network of individual Kindlins (GeneMANIA database). (B) Protein-protein interaction network of 716 individual Kindlins (STRING database). 

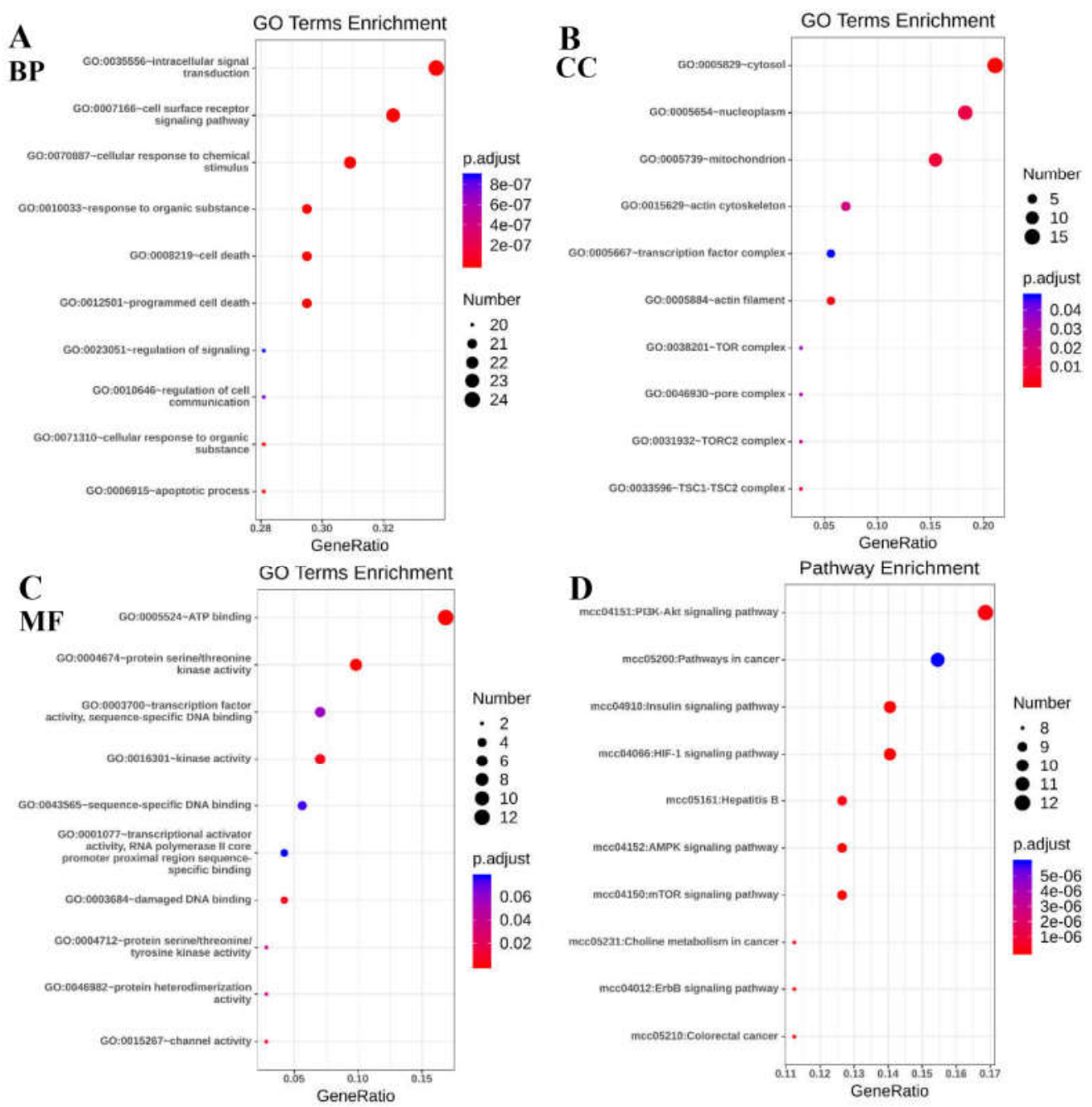

Fig.8. The functional enrichment analysis of Kindlins in Patients with NSCLC (DAVID).

Bubble chart of the top 10 results of GO Terms enrichment analysis with Biological

724 in the network; Bubble size: number of genes assigned to Go Terms function or KEGG pathway. Gene count and p-Values were considered to obtain important metabolic processes. 


\begin{tabular}{|c|c|c|c|c|c|c|}
\hline & Comparison groups & Fold & P value & t-test & Sample & Ref \\
\hline & & Change & & & size & \\
\hline \multirow[t]{5}{*}{ FERMT1 } & LUSC vs. Normal & 11.152 & $1.76 \mathrm{E}-24$ & 23.513 & 92 & Hou \\
\hline & LUAD vs. Normal & 3.430 & $1.57 \mathrm{E}-10$ & 7.780 & 110 & Hou \\
\hline & LUAD vs. Normal & 2.190 & $1.26 \mathrm{E}-6$ & 5.426 & 57 & $\mathrm{Su}$ \\
\hline & LUAD vs. Normal & 2.058 & 1.39E-11 & 9.585 & 246 & Okayama \\
\hline & LUSC vs. Normal & 2.993 & $2.20 \mathrm{E}-4$ & 7.925 & 10 & Wachi \\
\hline \multirow[t]{8}{*}{ FERMT2 } & LUAD vs. Normal & -2.029 & 5.71E-13 & -8.760 & 110 & Hou \\
\hline & LCC vs. Normal & -2.873 & $6.81 E-8$ & -7.776 & 84 & Hou \\
\hline & LUSC vs. Normal & -3.049 & $1.22 \mathrm{E}-10$ & -9.147 & 94 & Hou \\
\hline & LUAD vs. Normal & -2.571 & 5.08E-30 & -15.496 & 116 & Selamat \\
\hline & LUAD vs. Normal & -3.053 & 9.39E-11 & -8.536 & 57 & $\mathrm{Su}$ \\
\hline & LUAD vs. Normal & -2.417 & $6.56 \mathrm{E}-24$ & -13.005 & 107 & Landi \\
\hline & LUAD vs. Normal & -2.590 & $3.44 \mathrm{E}-8$ & -8.11 & 39 & Stearman \\
\hline & LUSC vs. Normal & -4.045 & $1.21 \mathrm{E}-4$ & -6.837 & 10 & Wachi \\
\hline FERMT3 & LCC vs. Normal & -3.541 & 8.33E-8 & -7.916 & 84 & Hou \\
\hline
\end{tabular}


728 LUAD, lung adenocarcinoma; LUSC, lung squamous cell carcinoma. LCC, lung large cell

729 carcinoma 
Figures
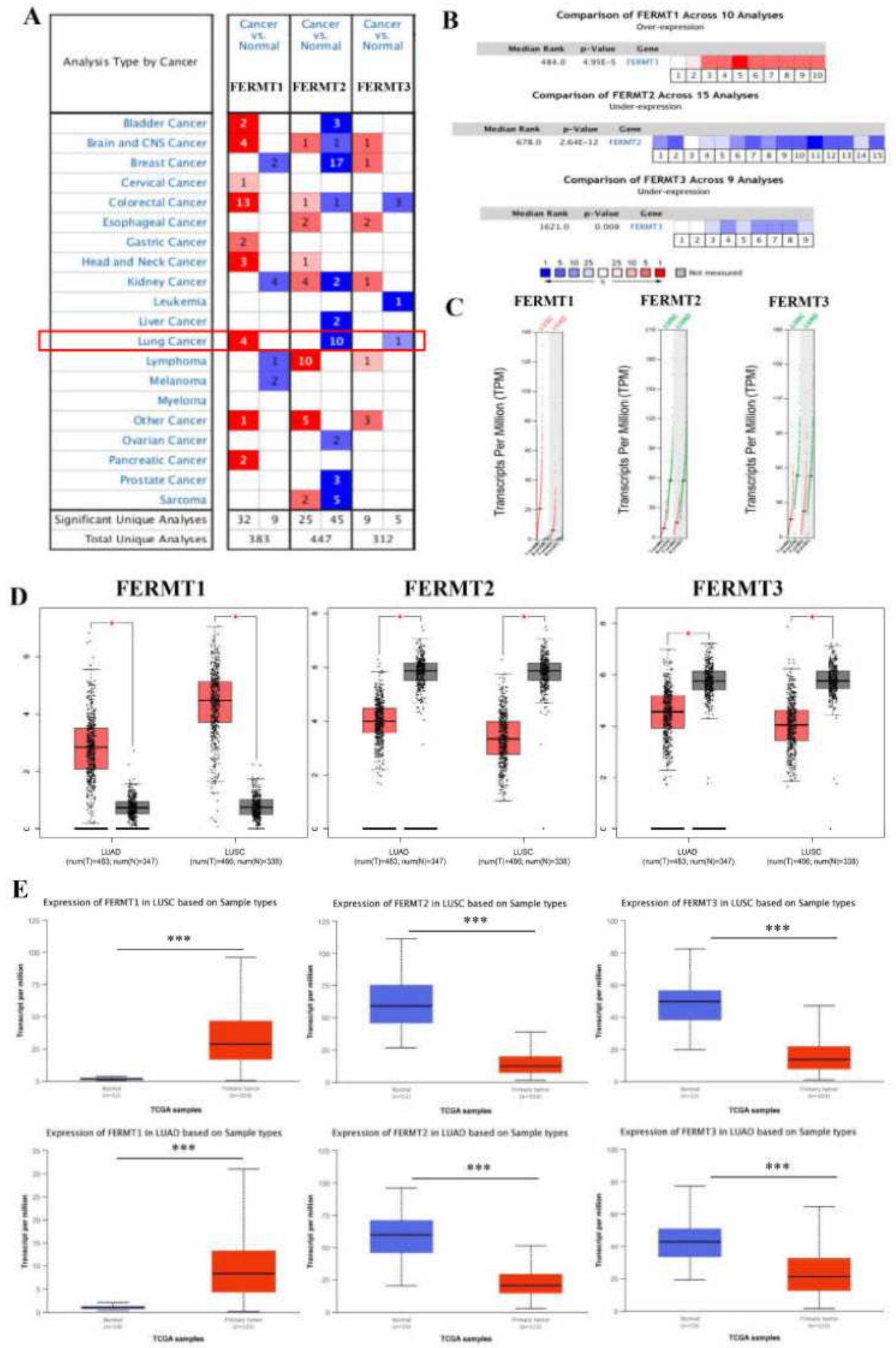

Figure 1

The expression of Kindlins in NSCLC. (A)The expression of Kindlins in different types of cancers compared with normal tissues (ONCOMINE). (B) Meta-analysis of FERMT1, FERMT2 and FERMT3 mRNA expression in NSCLC from multiple Oncomine databases. (C) The expression profile of Kindlins in LUAD 
and LUSD (GEPIA). Red trace, tumor samples; green trace, normal samples; T, tumor; N, normal. (D) The expression boxplots of Kindlins in LUSD and LUAD (GEPIA). A t-test was used to compare the expression level differences between tumor and normal tissues $(P<0.01)$. Y-axis represents log2 $(T P M+1)$. Red box, tumor samples; black box, normal samples. T, tumor; N, normal. (E) The relative expression of Kindlins in LUAD and LUSD (UALCAN).

A

FERMT1 mRNA expression (RNAseq)
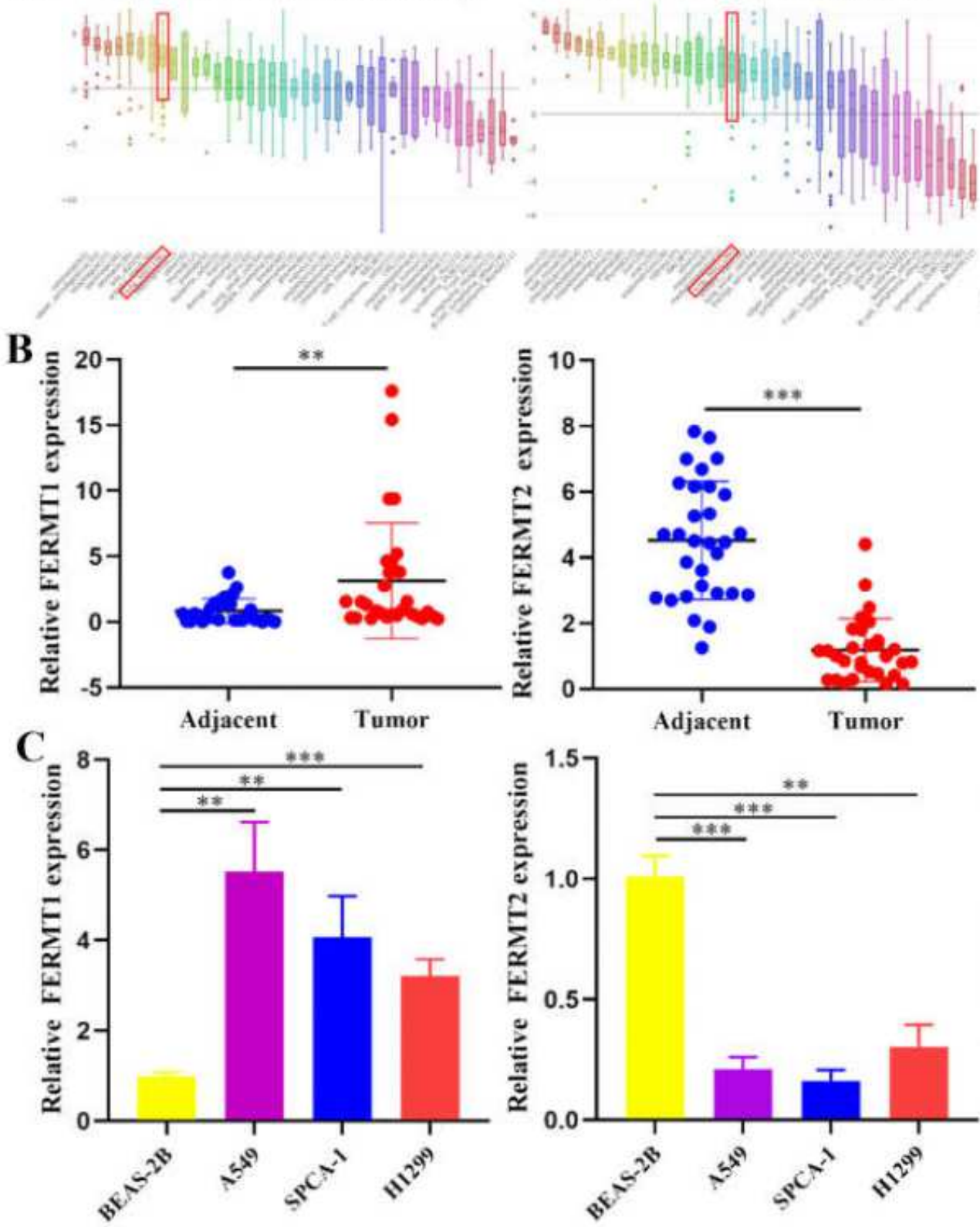

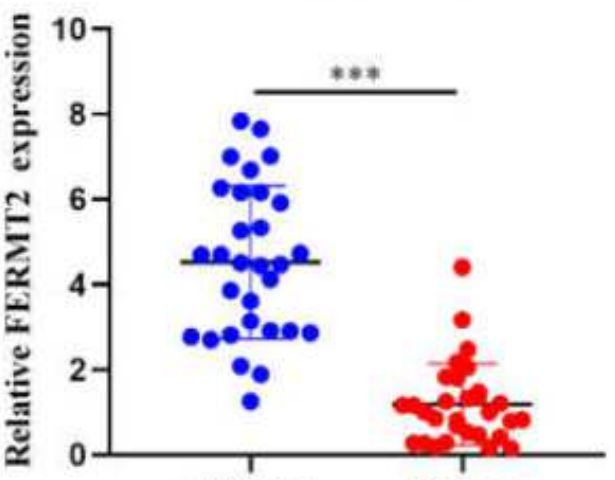

FERMT3 mRNA expression (RNAseq)
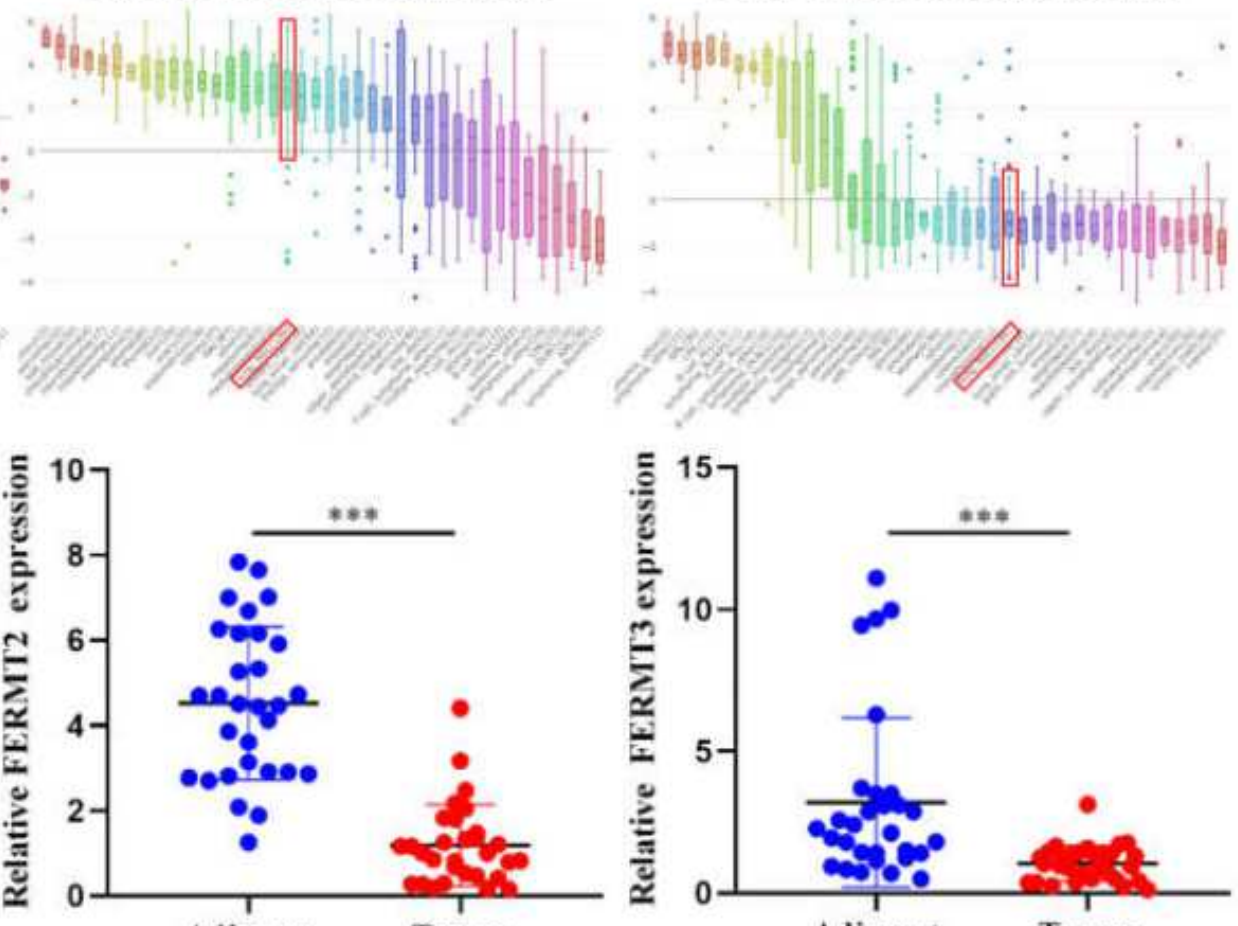

Adjacent

Tumor

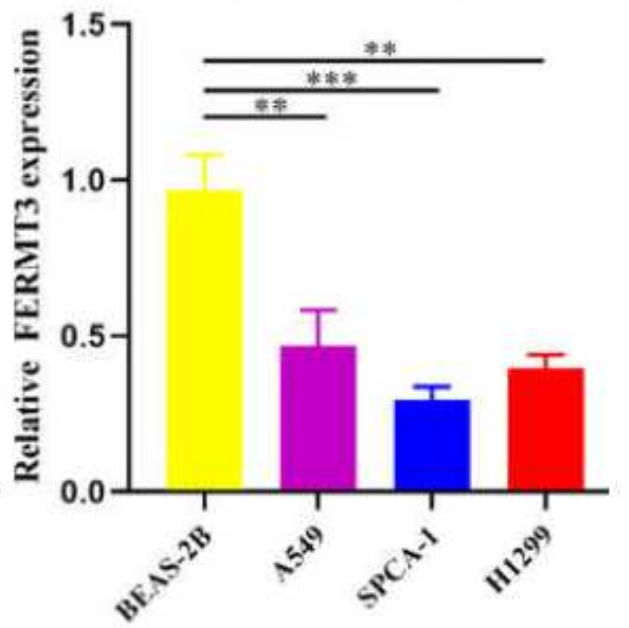

Figure 2

The expression of Kindlins in NSCLC tissue and cell lines. (A) FERMT1, FERMT2 and FERMT3 mRNA expression levels in NSCLC cell lines (CCLE). (B) Analysis of FERMT1, FERMT2 and FERMT3 expression in 30 pairs of NSCLC tissues and adjacent tissues in 41 NSCLC patients via RT-PCR assay. (C) Analysis of FERMT1, FERMT2 and FERMT3 expression in the NSCLC cell lines (A549, SPCA-1 and H1299) and one

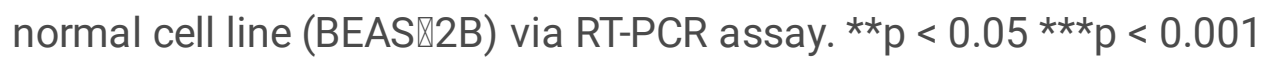



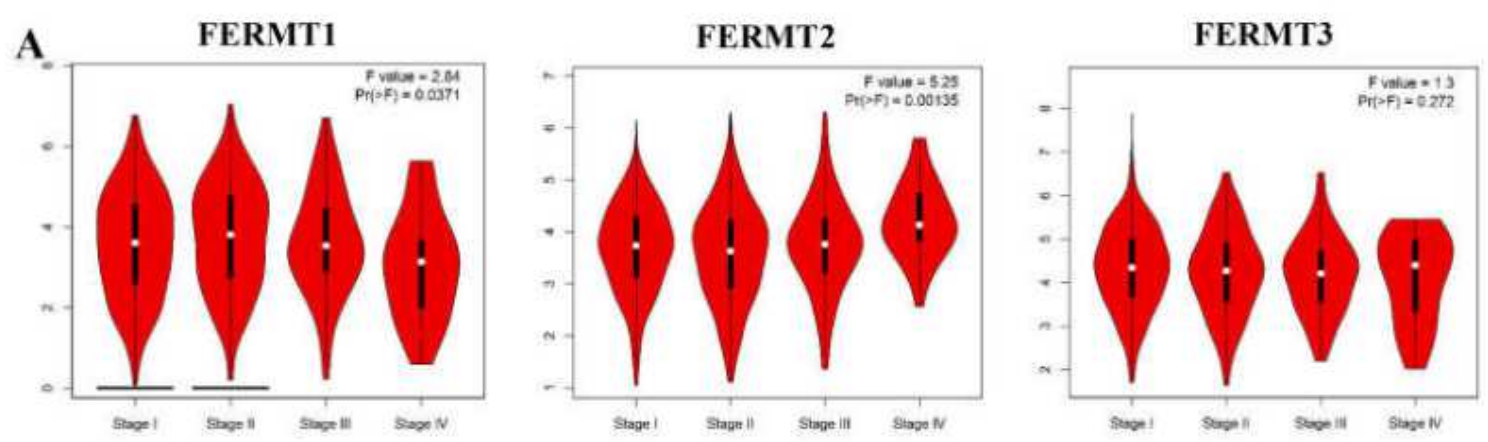

B Expression of HEMTr in Luap based an individual cancer
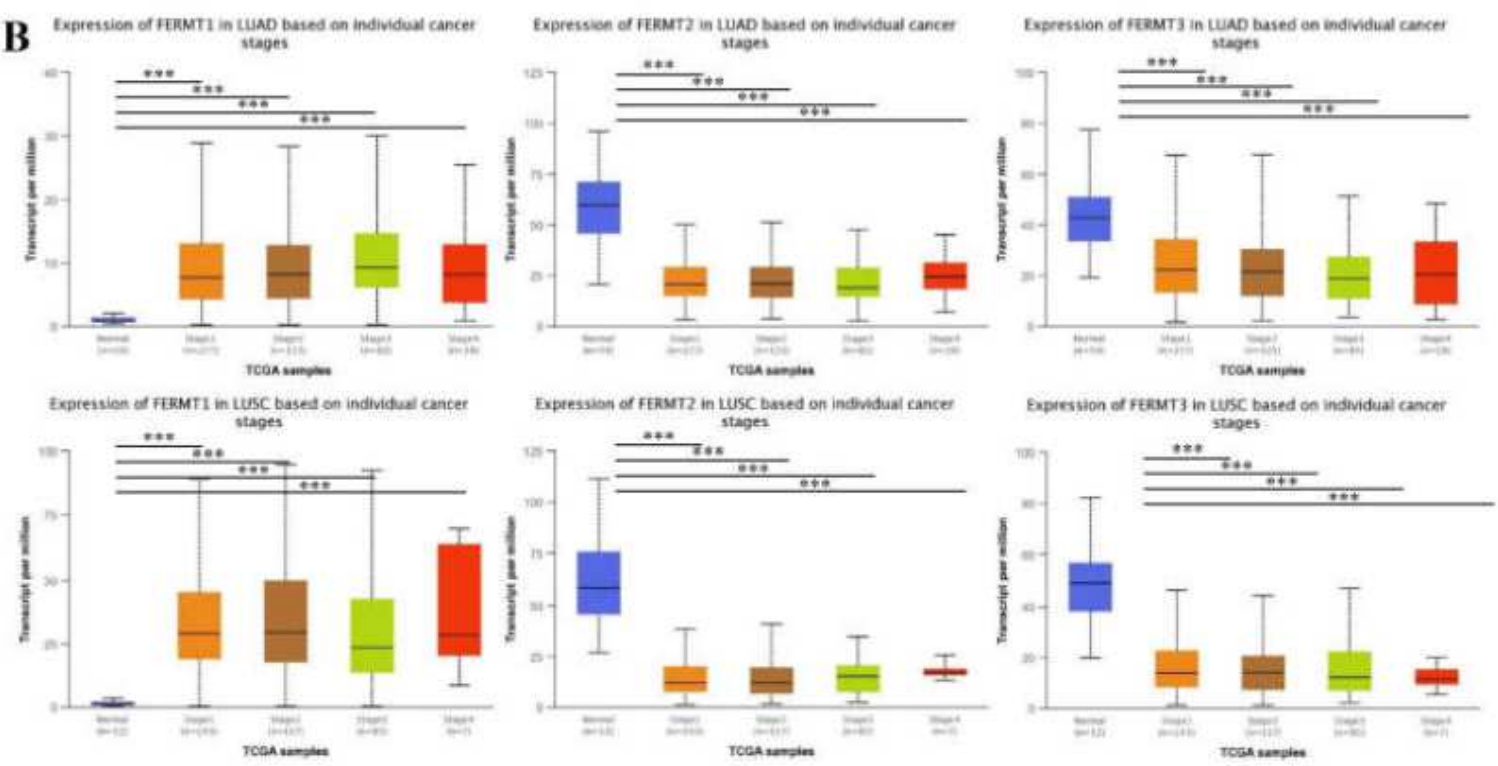

C
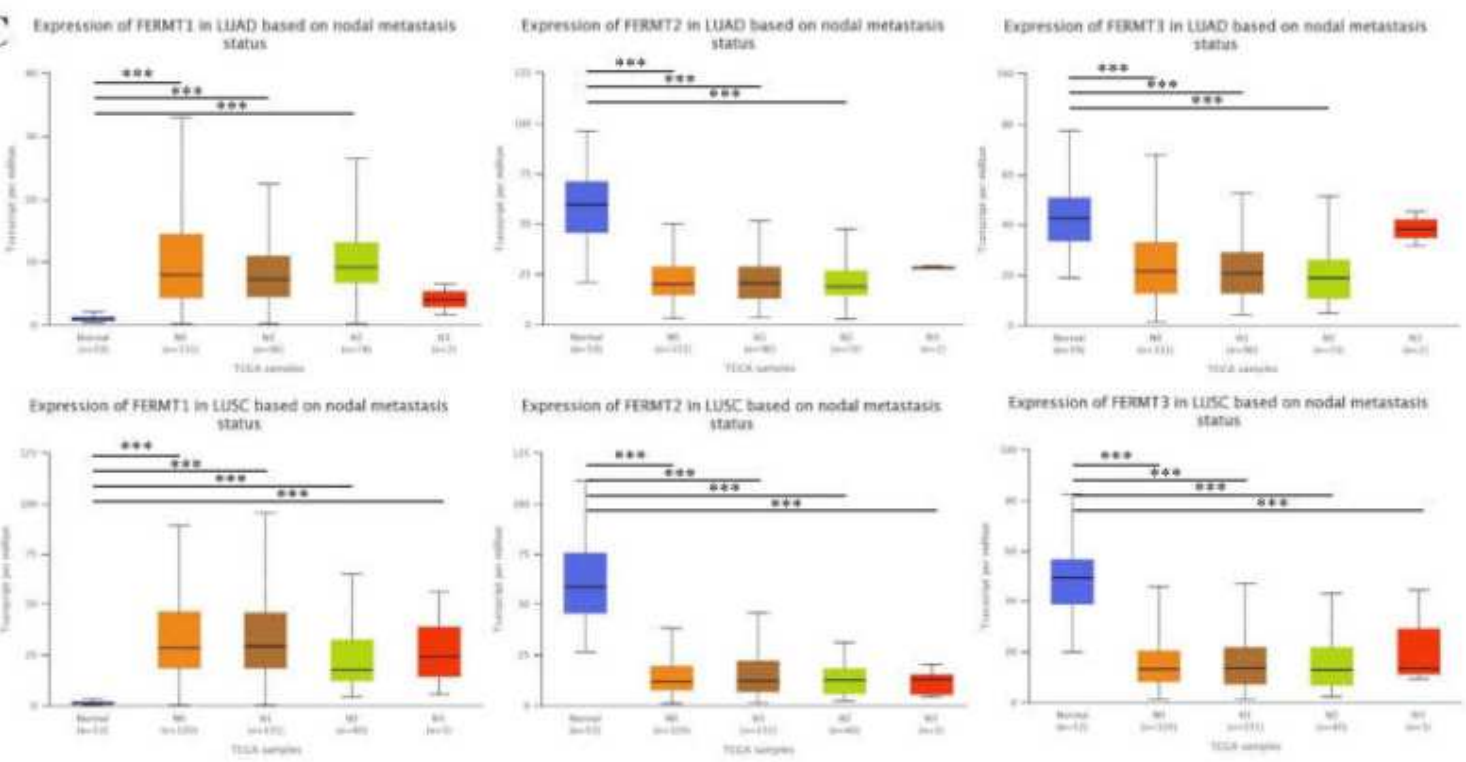

\section{Figure 3}

Association between the Kindlins expression and clinicopathological parameters in NSCLC. (A) Correlation between Kindlins expression and tumor stages in patients with NSCLC (GEPIA). (B) The correlations between Kindlins mRNA expression and clinicopathological stages in LUAD and LUSD (UALCAN). (C) The correlations between Kindlins mRNA expression and nodal metastasis status in LUAD

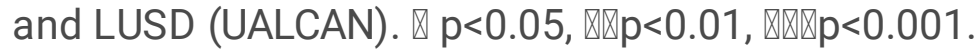




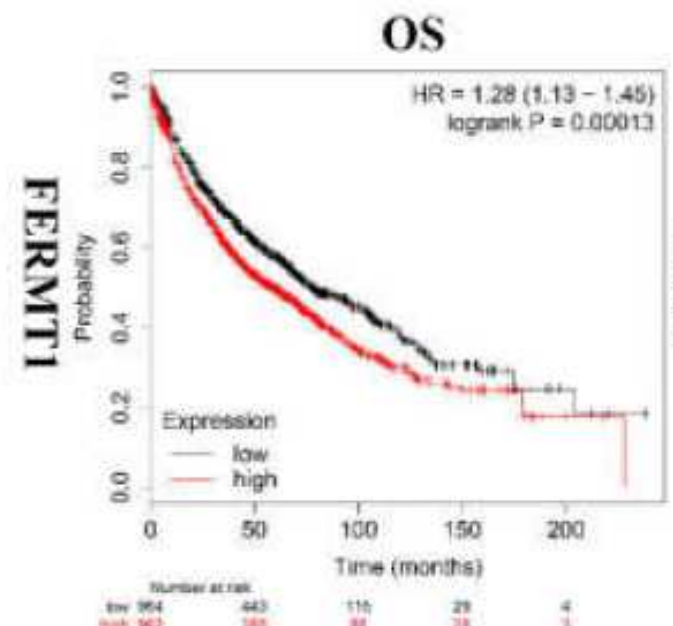

FP
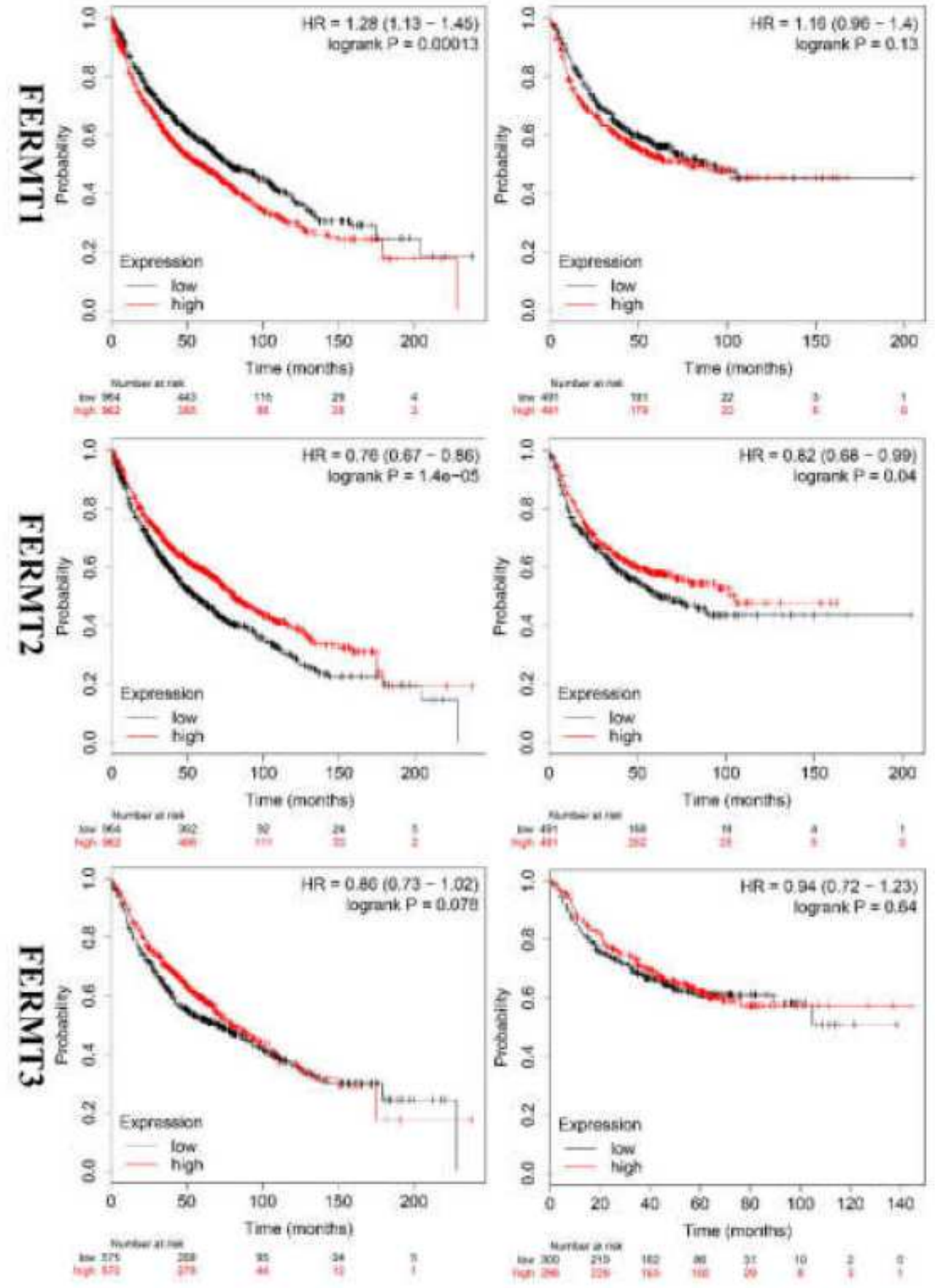
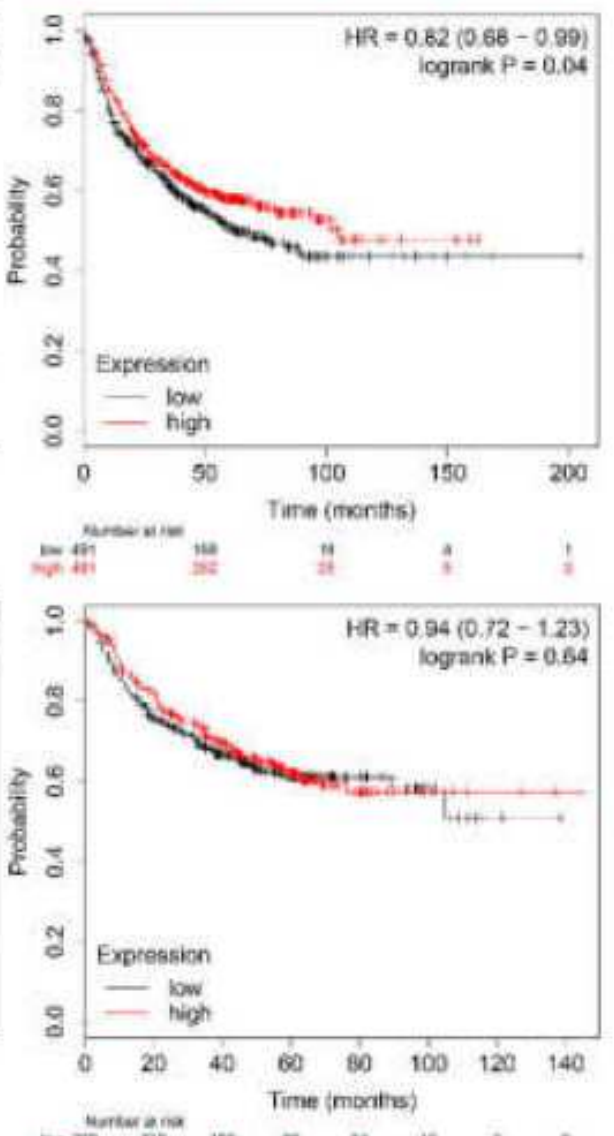

PPS
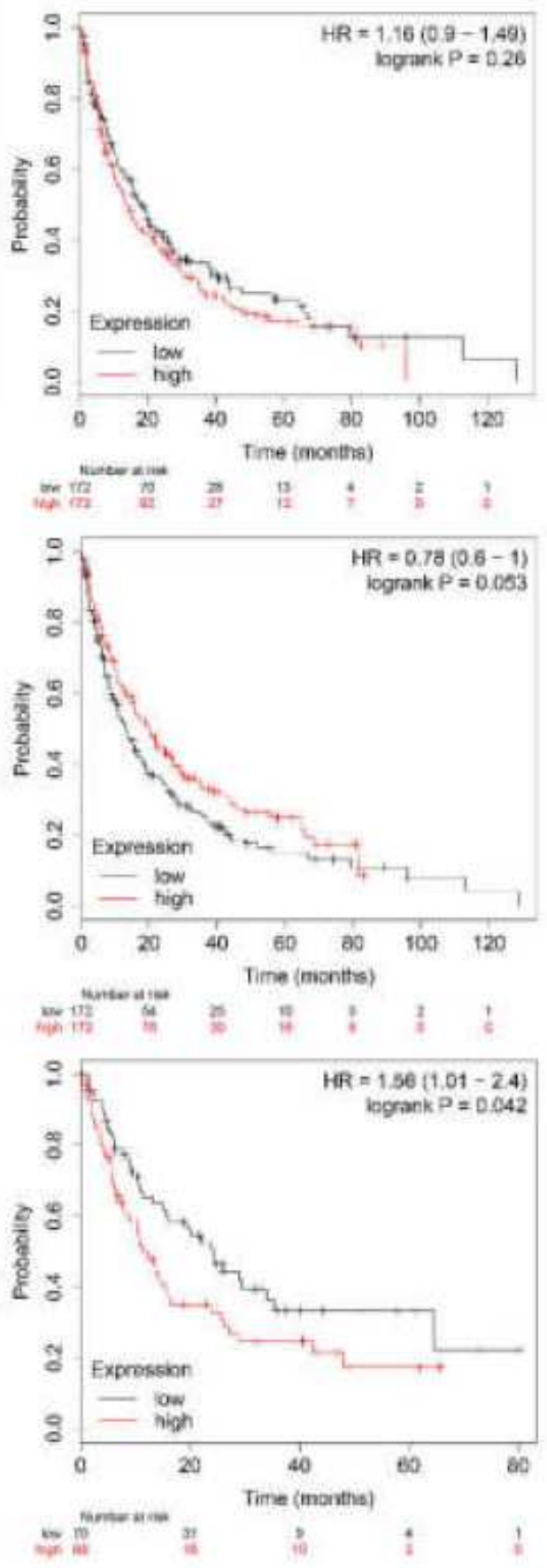

\section{Figure 4}

The prognostic value of the mRNA level of individual Kindlins in NSCLC patients (Kaplan-Meier plotter). OS, overall survival; FP, first progression; PPS, post-progression survival. 


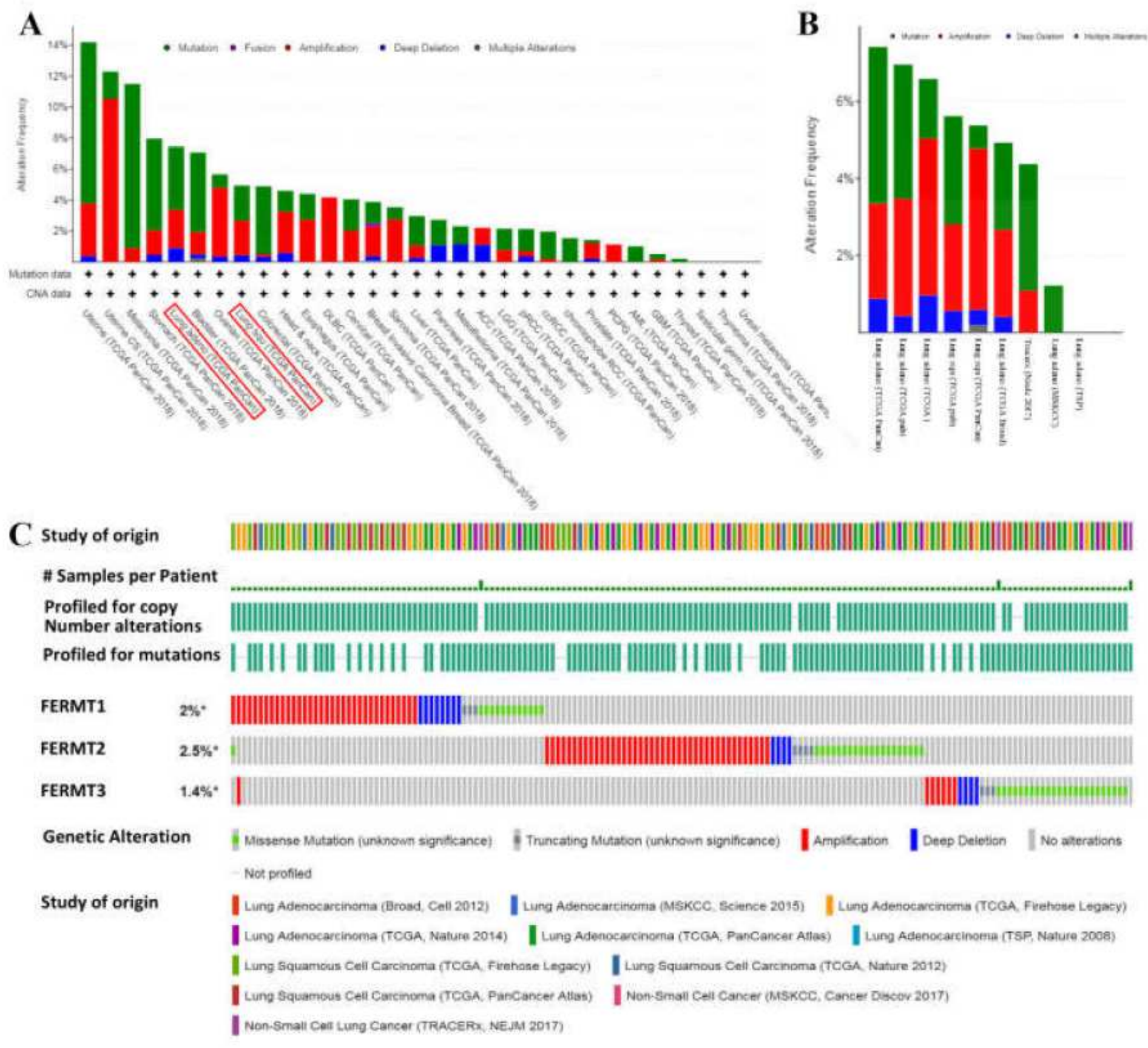

\section{Figure 5}

Analysis of Kindlins genetic alterations in NSCLC patients (cBioPortal). (A) Genetic alternations frequencies of Kindlin family members in various carcinoma types. Green, genetic mutations; purple, gene fusions; red, gene amplifications; blue, deep deletions; grey, multiple alterations. (B) Summary of Kindlin family genetic alterations in NSCLC. Alterations frequencies and alterations types of Kindlins were determined in 3897 patients / 4181 samples of 11 NSCLC studies. Green, genetic mutations; red, gene amplifications; blue, deep deletions; grey, multiple alterations. (C) OncoPrint visual summary of Kindlins alterations in NSCLC. green, missense mutations. gray, truncation mutation. Red, gene amplifications; blue, deep deletions. 

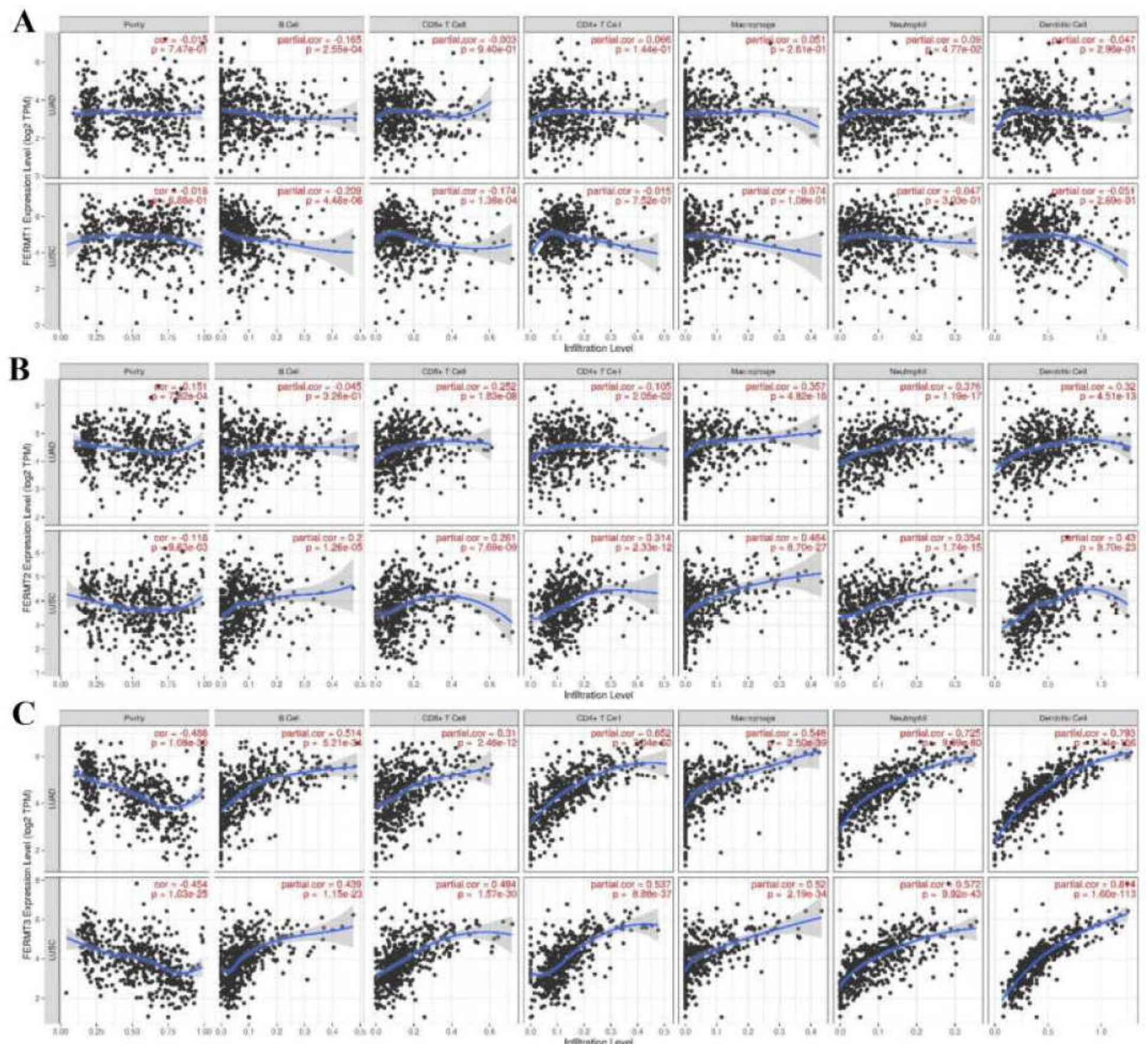

Figure 6

Association of Kindlins (FERMT1, FERMT2, and FERMT3) expression with immune infiltration of immune cells in LUAD and LUSD patients (TIMER). Correlation was analyzed using Pearson's Correlation. 


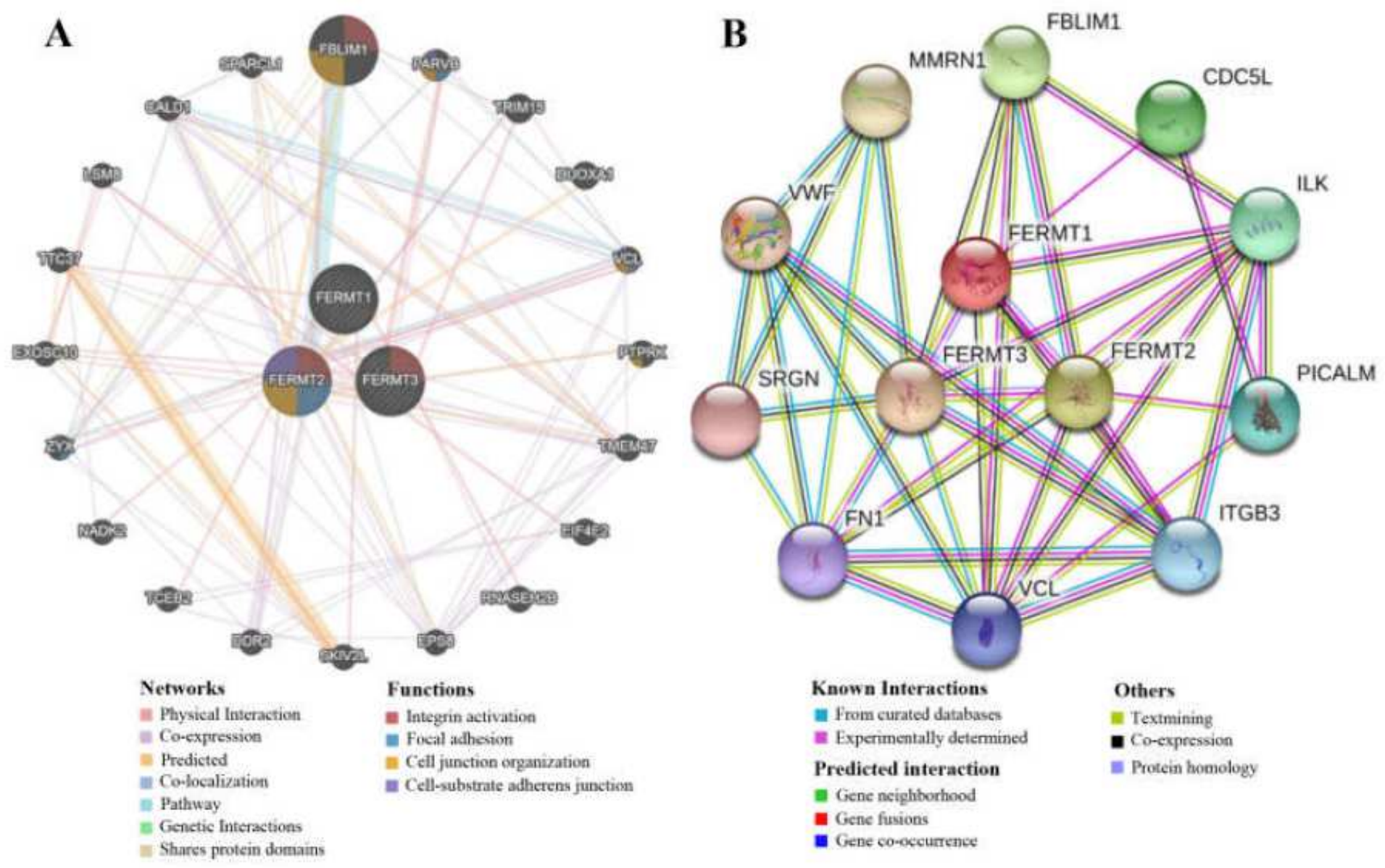

Figure 7

Interaction analysis of Kindlins family members. (A) Gene-gene interaction network of individual Kindlins (GeneMANIA database). (B) Protein-protein interaction network of individual Kindlins (STRING database). 


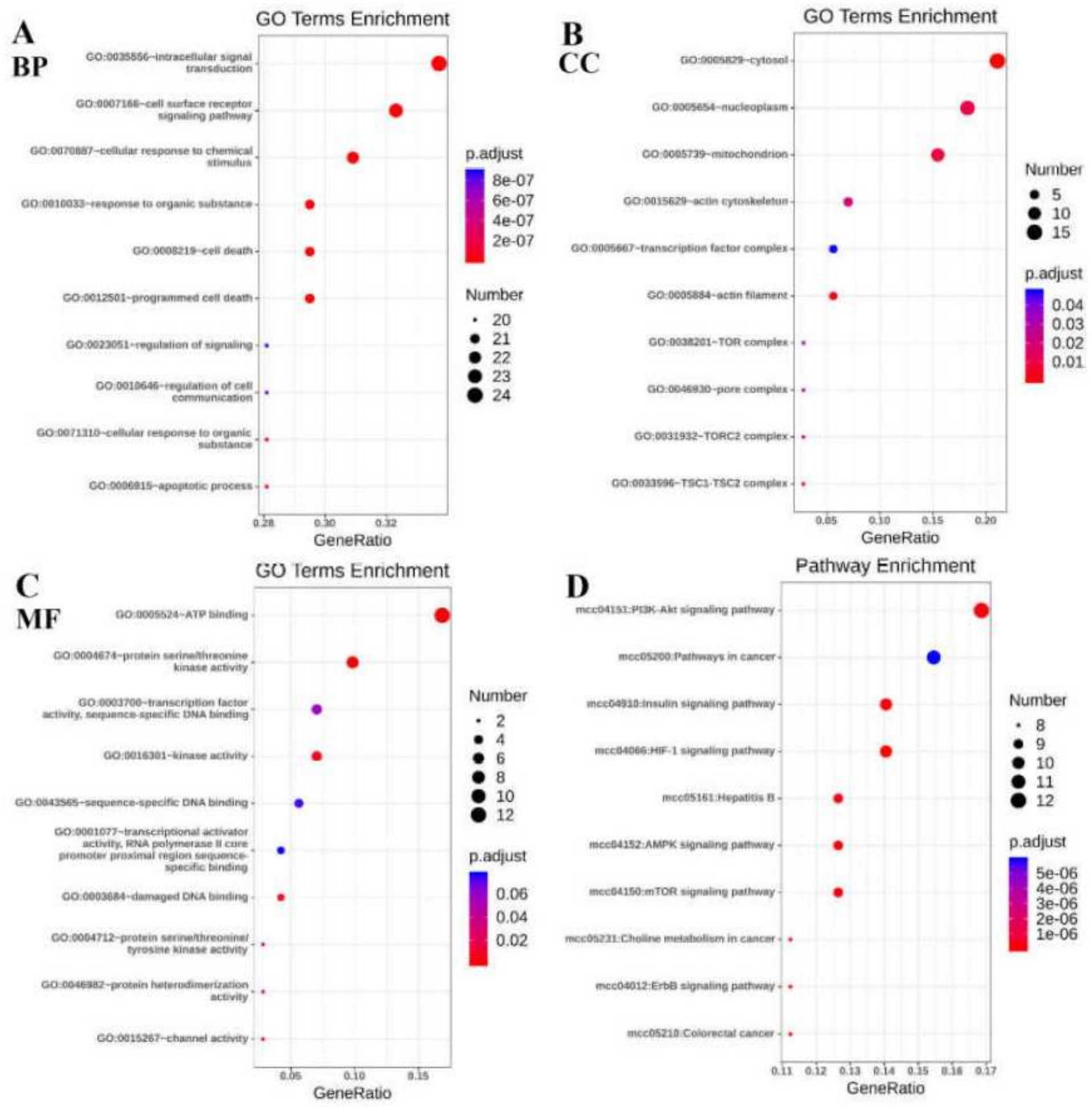

Figure 8

The functional enrichment analysis of Kindlins in Patients with NSCLC (DAVID). Bubble chart of the top 10 results of $\mathrm{GO}$ Terms enrichment analysis with Biological Process(A), Cellular Component (B) and Molecular Function (C). (D) Bubble chart of the top 10 results of KEGG pathway enrichment analysis. Yaxis: name of the Go Terms function or KEGG pathway; X-axis: percentage of genes assigned to a term in the total number of genes in the network; Bubble size: number of genes assigned to Go Terms function or KEGG pathway. Gene count and p-Values were considered to obtain important metabolic processes. 


\section{Supplementary Files}

This is a list of supplementary files associated with this preprint. Click to download.

- SupplementaryTable1..doc

- Supplementarymaterial2Proteomicdata.txt

- Supplementarymaterial3GOBiologicalProcess.txt

- Supplementarymaterial4GOCellularComponent.txt

- Supplementarymaterial5GOMolecularFunction.txt

- Supplementarymaterial6KEGGpathway.txt 